\title{
SECTION 4: INVESTIGATION OF POTENTIAL SOURCES OF VARIATION
}

\subsection{INTRODUCTION}

The design of FIRI is such that for each laboratory, we have some basic, though limited, information on the laboratory procedures, including the method of pretreatment applied to the samples, the modern standard, and the background material used. These can be considered as factors in the experiment and through statistical analysis, we can investigate whether they offer a statistically significant explanation of the observed variation. The different levels of the factors are described in Table 4.1. In addition, the laboratory type is also considered as a further factor (with 3 levels of LSC, GPC, and AMS).

Therefore, this section considers each sample, in turn, and explores the proportion of variation, which can be explained by each of the factors. For these analyses, extreme values (outliers) have been omitted (as identified in Section 3).

The structure of the section for each sample includes the summary statistics with the number of omitted values from the analysis, a boxplot showing the distribution of the results for the different levels of the factor of interest, and the output from a formal analysis of variance (a formal test of the hypothesis that the mean age/activity is the same for each level of the factor). This output takes the form of a table, where the key statistic is the $p$-value. Conventionally, at a 5\% significance level, if the p-value is less than 0.05 , then we reject that the mean age/activity is the same for all levels of the factor and conclude there are statistically significant differences. In such a case, a follow-up analysis can be used to identify the magnitude of any differences.

Table 4.1 Classifications used for modern standard and background material

\begin{tabular}{ll}
\hline a) Modern standard & \\
\hline Original description & Analysis classification/level \\
\hline ANU sucrose & ASuc \\
Benzene & Benz(ene) \\
NIST OxI & NBS1 \\
NIST OxII & NBS2 \\
GIN/HD-95,C-3 & Other \\
NIST I/II & NBS12 \\
& \\
b) Background & \\
\hline Original description & Analysis classification \\
\hline Anthracite & Anth(racite) \\
Benzene & Benz(ene) \\
Calcite & Calc(ite) \\
Charcoal & Charc(oal) \\
Bituminous coal & Coal \\
Graphite & Graphite \\
Doublespar/IAEA C1 & Marble \\
IAEA C4/wood/limestone & Other \\
\hline
\end{tabular}

\subsection{LABORATORY TYPE AS A SOURCE OF VARIATION}

In this section, the analysis is focused on whether there are statistically significant differences in the mean activity/age among the different laboratory types. 


\subsubsection{Sample C: Turbidite}

Table 4.2 Summary statistics of age for Sample C

\begin{tabular}{llrlll}
\hline Type & $\mathrm{N}$ & Nr of omitted values & Mean & Median & StDev \\
\hline AMS & 34 & 0 & 18,175 & 18,175 & 135 \\
GPC & 17 & 1 & 18,152 & 18,200 & 288 \\
LSC & 33 & 10 & 18,110 & 18,120 & 244 \\
AMS & 34 & 0 & 18,175 & 18,175 & 135 \\
\hline
\end{tabular}

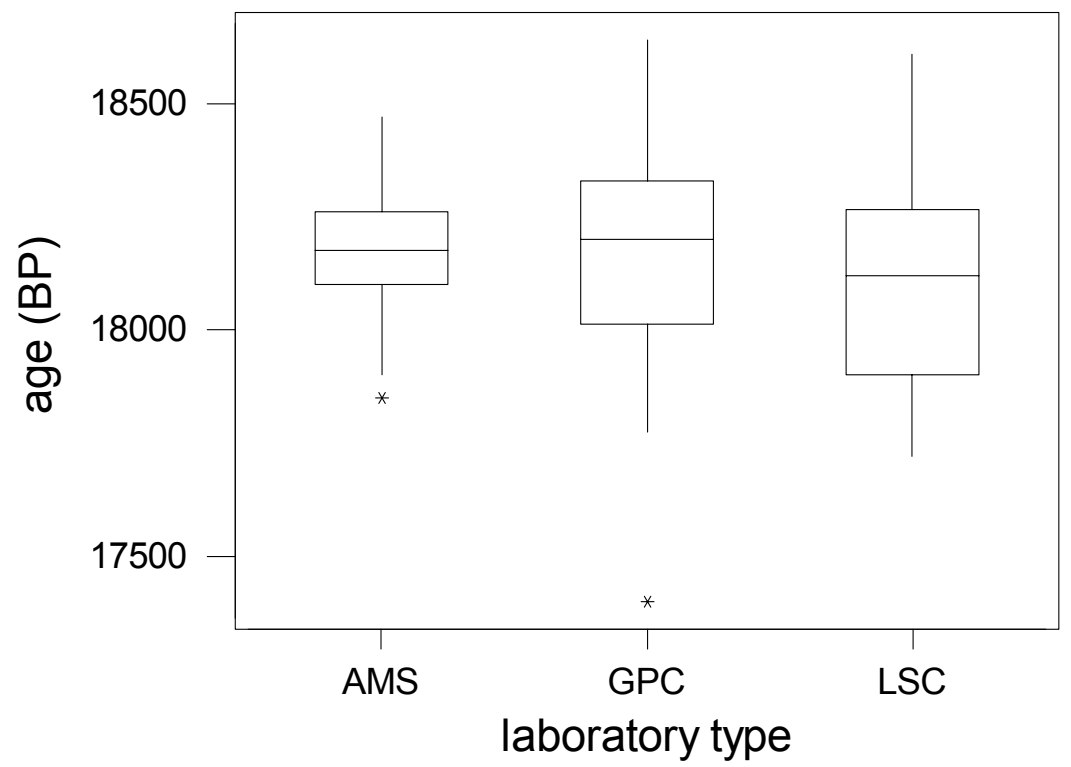

Figure 4.1 Distribution of age by laboratory type for Sample C

Table 4.3 Analysis of variance for Sample C

\begin{tabular}{|c|c|c|c|c|}
\hline Source & $\mathrm{DF}$ & SS & MS & F \\
\hline type & 2 & 70472 & 35236 & 0.478 \\
\hline Error & 81 & 3830128 & 47286 & \\
\hline \multirow[t]{2}{*}{ Total } & 83 & 3900600 & & \\
\hline & & & & $\begin{array}{l}\text { Individual 95\% CIs For Mean } \\
\text { Based on Pooled StDev }\end{array}$ \\
\hline Level & $\mathrm{N}$ & Mean & StDev & -----+-----------+----------++----------+- \\
\hline AMS & 34 & 18175 & 135 & $(-----------\star *-----------)$ \\
\hline GPC & 17 & 18152 & 288 & $(-----------------\star------------------)$ \\
\hline LSC & 33 & 18110 & 244 & $(-----------\star------------)$ \\
\hline Pooled & $\mathrm{eV}=$ & 217 & & 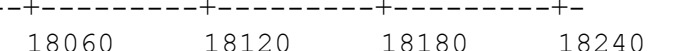 \\
\hline
\end{tabular}

\subsubsection{Conclusion}

Since the p-value is $>0.05$ in Table 4.3 , there is no evidence of statistically significant differences among laboratory types. The results from each laboratory type broadly overlap. 


\subsubsection{Sample E: Humic Acid}

Table 4.4 Summary statistics of age for Sample E

\begin{tabular}{llllll}
\hline Type & $\mathrm{N}$ & Number omitted & Mean & Median & StDev \\
\hline AMS & 64 & 1 & 11,804 & 11,800 & 117 \\
GPC & 23 & 4 & 11,743 & 11,722 & 173 \\
LSC & 38 & 14 & 11,757 & 11,736 & 177 \\
\hline
\end{tabular}

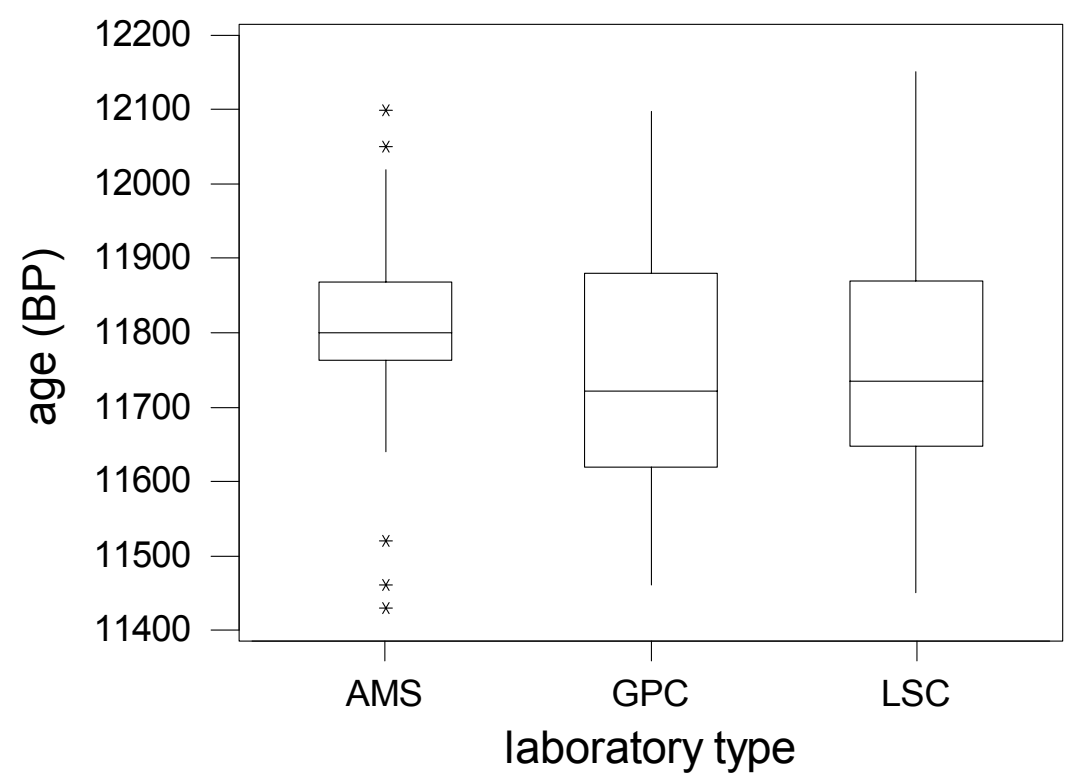

Figure 4.2 Distribution of age by laboratory type for Sample E

Table 4.5 Analysis of variance for Sample E

\begin{tabular}{|c|c|c|c|c|}
\hline Source & $\mathrm{DF}$ & SS & MS & F \\
\hline type & 2 & 86788 & 43394 & 0.143 \\
\hline Error & 122 & 2680561 & 21972 & \\
\hline Total & 124 & 2767349 & & \\
\hline & & & & $\begin{array}{l}\text { Individual 95\% CIs For Mean } \\
\text { Based on Pooled StDev }\end{array}$ \\
\hline Level & $\mathrm{N}$ & Mean & StDev & ----+----------+----------+----------+-- \\
\hline AMS & 64 & 11804 & 117 & $(-------\star------)$ \\
\hline GPC & 23 & 11743 & 173 & $(------------\star-----------)$ \\
\hline LSC & 38 & 11757 & 177 & $(--------\star---------)$ \\
\hline
\end{tabular}

\subsubsection{Conclusion}

Since the p-value is $>0.05$ in Table 4.5 , there is no evidence of statistically significant differences among laboratory types. The results from each laboratory type broadly overlap. 


\subsubsection{Sample D: Belfast Wood}

Table 4.6 Summary statistics of age for Sample D

\begin{tabular}{|c|c|c|c|c|c|}
\hline Type & $\mathrm{N}$ & Number omitted & Mean & Median & StDev \\
\hline AMS & 41 & 0 & 4530.3 & 4520.0 & 52.0 \\
\hline GPC & 19 & 1 & 4506.7 & 4509.0 & 56.5 \\
\hline LSC & 38 & 12 & 4521.7 & 4537.5 & 106.7 \\
\hline
\end{tabular}

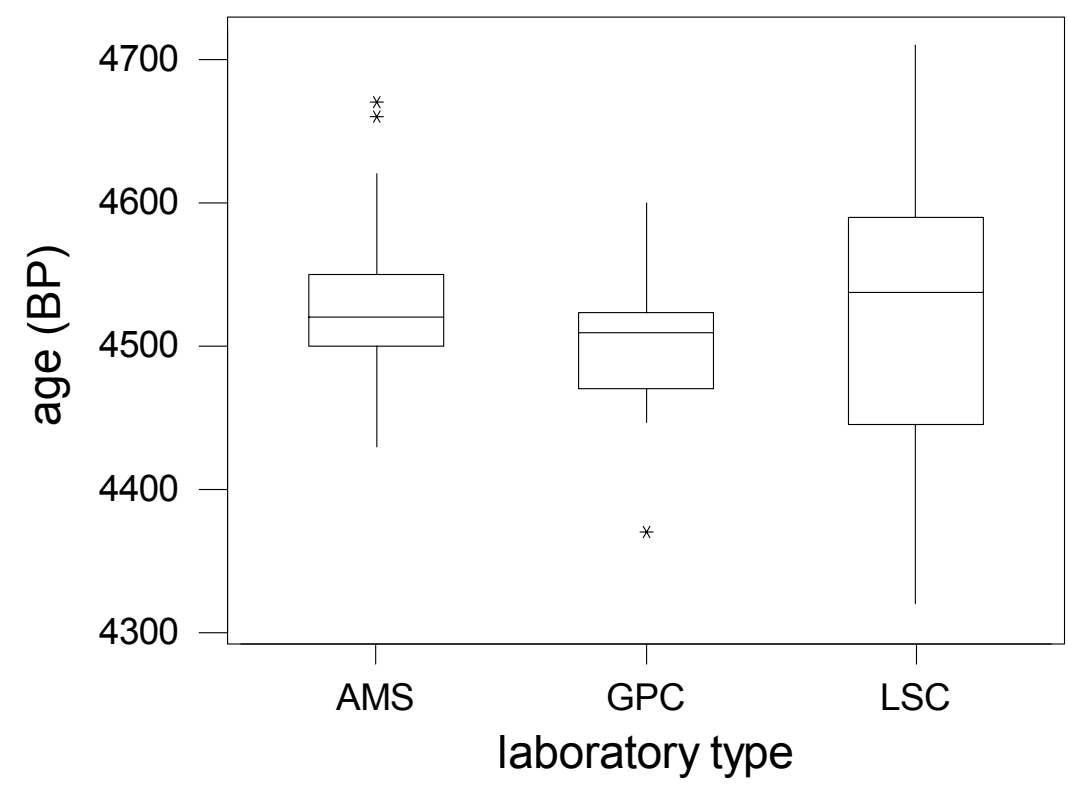

Figure 4.3 Distribution of age by laboratory type for Sample D

Table 4.7 Analysis of variance of Sample D

\begin{tabular}{|c|c|c|c|c|}
\hline Source & $\mathrm{DF}$ & SS & $\mathrm{MS}$ & F \\
\hline type & 2 & 7231 & 3616 & 0.559 \\
\hline Error & 95 & 586712 & 6176 & \\
\hline \multirow[t]{2}{*}{ Total } & 97 & 593943 & & \\
\hline & & & & $\begin{array}{l}\text { Individual 95\% CIs For Mean } \\
\text { Based on Pooled StDev }\end{array}$ \\
\hline Level & $\mathrm{N}$ & Mean & StDev & --+---------+---------+---------+---- \\
\hline AMS & 41 & 4530.3 & 52.0 & $(---------*---------)$ \\
\hline GPC & 19 & 4506.7 & 56.5 & $(--------------\star-------------)$ \\
\hline LSC & 38 & 4521.7 & 106.7 & $(---------\star---------)$ \\
\hline Pooled & StDev $=$ & 78.6 & & 4475 \\
\hline
\end{tabular}

\subsubsection{Conclusion}

Since the p-value is $>0.05$ in Table 4.7 , there is no evidence of statistically significant differences among laboratory types. The results from each laboratory type broadly overlap. 


\subsubsection{Sample F: Belfast Wood}

Table 4.8 Summary statistics of age for Sample F

\begin{tabular}{llclll}
\hline Type & $\mathrm{N}$ & Number omitted & Mean & Median & StDev \\
\hline AMS & 37 & 0 & 4534.2 & 4534.0 & 62.0 \\
GPC & 18 & 3 & 4495.3 & 4476.5 & 85.4 \\
LSC & 35 & 13 & 4493.8 & 4500.0 & 83.4 \\
\hline
\end{tabular}

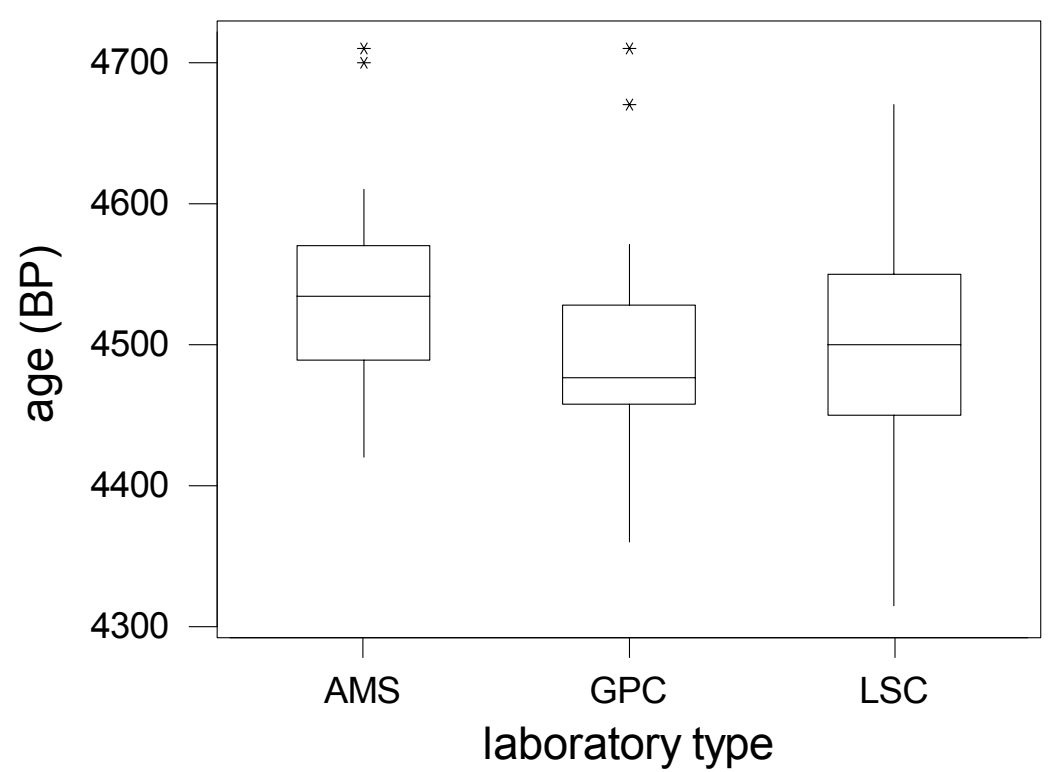

Figure 4.4 Distribution of age by laboratory type for Sample F

Table 4.9 Analysis of variance for Sample F

\begin{tabular}{|c|c|c|c|c|}
\hline Source & $\mathrm{DF}$ & SS & MS & $\mathrm{F}$ \\
\hline type & 2 & 34805 & 17402 & 0.053 \\
\hline Error & 87 & 499422 & 5740 & \\
\hline \multirow[t]{3}{*}{ Total } & 89 & 534227 & & \\
\hline & & & & Individual 95\% CIs For Mean \\
\hline & & & & Based on Pooled StDev \\
\hline Level & $\mathrm{N}$ & Mean & StDev & ----+---------+---------+---------+-- \\
\hline AMS & 37 & 4534.2 & 62.0 & $(-------\star--------)$ \\
\hline GPC & 18 & 4495.3 & 85.4 & $(----------*-----------)$ \\
\hline \multirow[t]{2}{*}{$\mathrm{LSC}$} & 35 & 4493.8 & 83.4 & $(--------\star-------)$ \\
\hline & & & & -----+--- \\
\hline Pooled & StDev $=$ & 75.8 & & 4530 \\
\hline
\end{tabular}

\subsubsection{Conclusion}

A statistically significant difference among laboratory types is detected at the $10 \%$ level ( $p$-value $=$ 0.053 in Table 4.9). The mean age from AMS laboratories appears older than that for either GPC or LSC laboratories. 


\subsubsection{Sample G: Barley Mash (pMC)}

Table 4.10 Summary statistics of activity (pMC) for Sample G

\begin{tabular}{llllll}
\hline Type & $\mathrm{N}$ & Number omitted & Mean & Median & StDev \\
\hline AMS & 34 & 0 & 110.33 & 110.35 & 0.68 \\
GPC & 18 & 1 & 110.85 & 111.00 & 1.06 \\
LSC & 32 & 15 & 110.53 & 110.60 & 1.10 \\
\hline
\end{tabular}

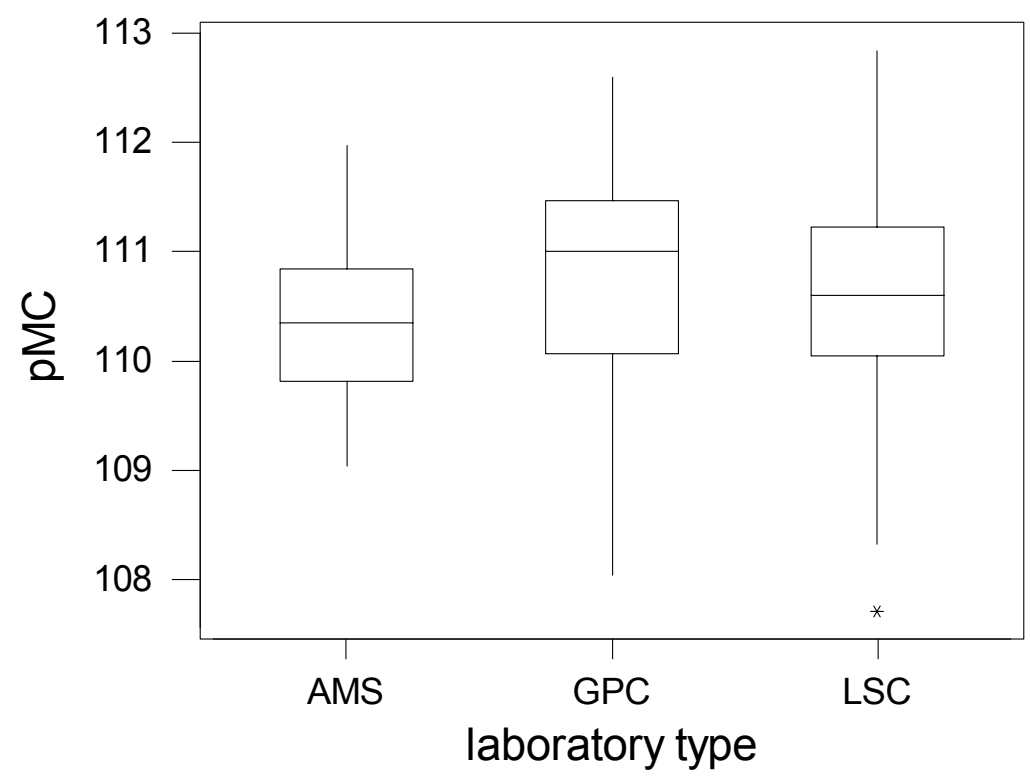

Figure 4.5 Distribution of activity by laboratory type for Sample G

Table 4.11 Analysis of variance for Sample G

\begin{tabular}{|c|c|c|c|c|}
\hline Source & $\mathrm{DF}$ & SS & MS & F \\
\hline type & 2 & 3.154 & 1.577 & 0.178 \\
\hline Error & 81 & 72.530 & 0.895 & \\
\hline \multirow[t]{2}{*}{ Total } & 83 & 75.684 & & \\
\hline & & & & $\begin{array}{l}\text { Individual 95\% CIs For Mean } \\
\text { Based on Pooled StDev }\end{array}$ \\
\hline Level & $\mathrm{N}$ & Mean & StDev & -------+---------+---------+--------- \\
\hline AMS & 34 & 110.335 & 0.685 & $(--------\star---------)$ \\
\hline GPC & 18 & 110.852 & 1.064 & $(-----------\star \star------------)$ \\
\hline LSC & 32 & 110.526 & 1.105 & $(---------\star--------)$ \\
\hline Pooled & StDev $=$ & 0.946 & & 110.95 \\
\hline
\end{tabular}

\subsubsection{Conclusion}

Since the p-value is $>0.05$ in Table 4.11 , there is no evidence of statistically significant differences among laboratory types. The results from each laboratory type broadly overlap. 


\subsubsection{Sample J: Barley Mash}

Table 4.12 Summary statistics of activity (pMC) for Sample J

\begin{tabular}{llllll}
\hline Type & $\mathrm{N}$ & Number omitted & Mean & Median & StDev \\
\hline AMS & 35 & 0 & 110.58 & 110.56 & 0.61 \\
GPC & 18 & 1 & 110.60 & 110.70 & 0.83 \\
LSC & 34 & 12 & 110.63 & 110.84 & 1.20 \\
\hline
\end{tabular}

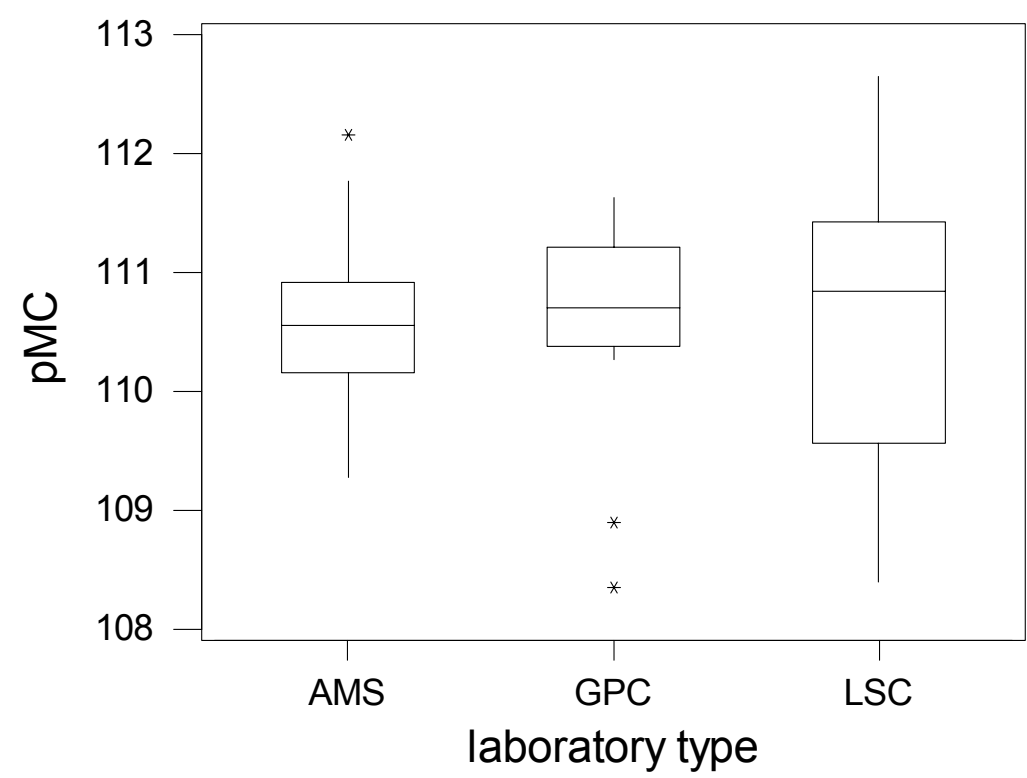

Figure 4.6 Distribution of activity by laboratory type for Sample J

Table 4.13 Analysis of variance for Sample J

\begin{tabular}{|c|c|c|c|c|}
\hline Source & $\mathrm{DF}$ & SS & MS & $\mathrm{P}$ \\
\hline type & 2 & 0.047 & 0.024 & 0.973 \\
\hline Error & 84 & 71.700 & 0.854 & \\
\hline \multirow[t]{3}{*}{ Total } & 86 & 71.748 & & \\
\hline & & & & Individual 95\% CIs For Mean \\
\hline & & & & Based on Pooled StDev \\
\hline Level & $\mathrm{N}$ & Mean & StDev & ----+---------+---------+---------+-- \\
\hline AMS & 35 & 110.583 & 0.606 & $(-----------*------------)$ \\
\hline GPC & 18 & 110.600 & 0.834 & $(----------------\star----------------)$ \\
\hline \multirow[t]{2}{*}{$\mathrm{LSC}$} & 34 & 110.635 & 1.198 & $(-----------\star------------)$ \\
\hline & & & & ----+---------+---------+---------+-- \\
\hline Pooled & StDev $=$ & 0.924 & & 111.00 \\
\hline
\end{tabular}

\subsubsection{Conclusion}

Since the p-value is $>0.05$ in Table 4.13 , there is no evidence of statistically significant differences among laboratory types. The results from each laboratory type broadly overlap. 


\subsubsection{Sample H: Hohenheim Wood}

Table 4.14 Summary statistics of age for Sample H

\begin{tabular}{llllll}
\hline Type & $\mathrm{N}$ & Number omitted & Mean & Median & StDev \\
\hline AMS & 36 & 0 & 2228.7 & 2230.0 & 48.2 \\
GPC & 19 & 1 & 2221.7 & 2200.0 & 95.4 \\
LSC & 33 & 13 & 2233.7 & 2230.0 & 98.1 \\
\hline
\end{tabular}

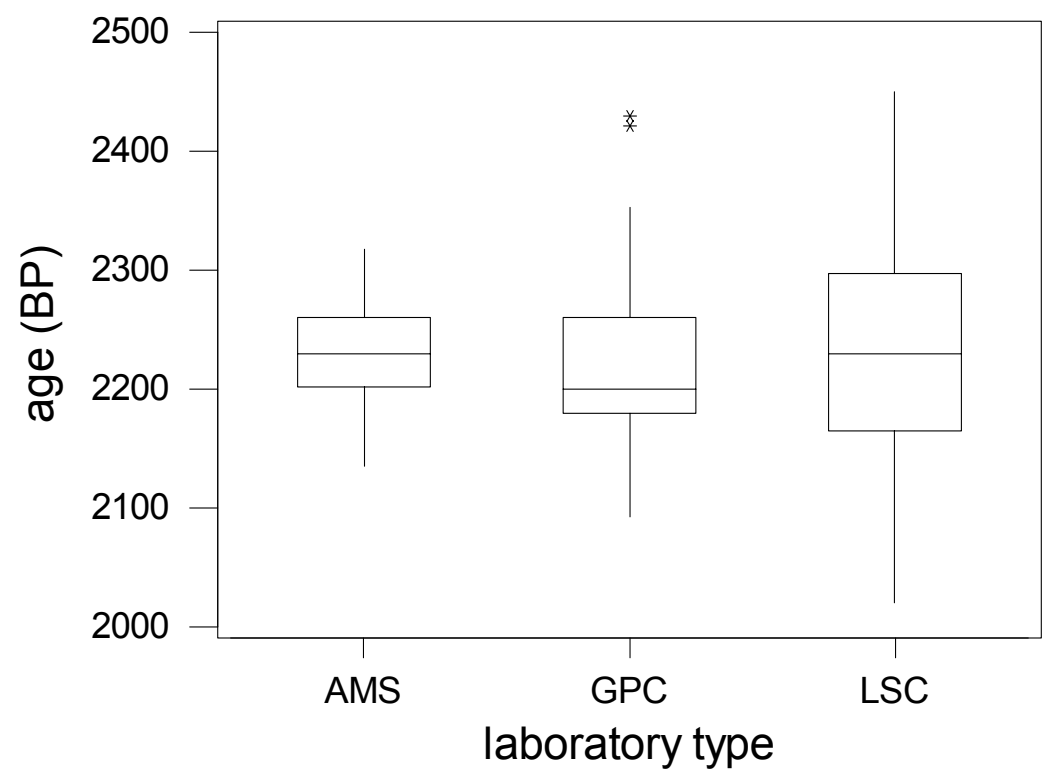

Figure 4.7 Distribution of age by laboratory type for Sample H

Table 4.15 Analysis of variance for Sample H

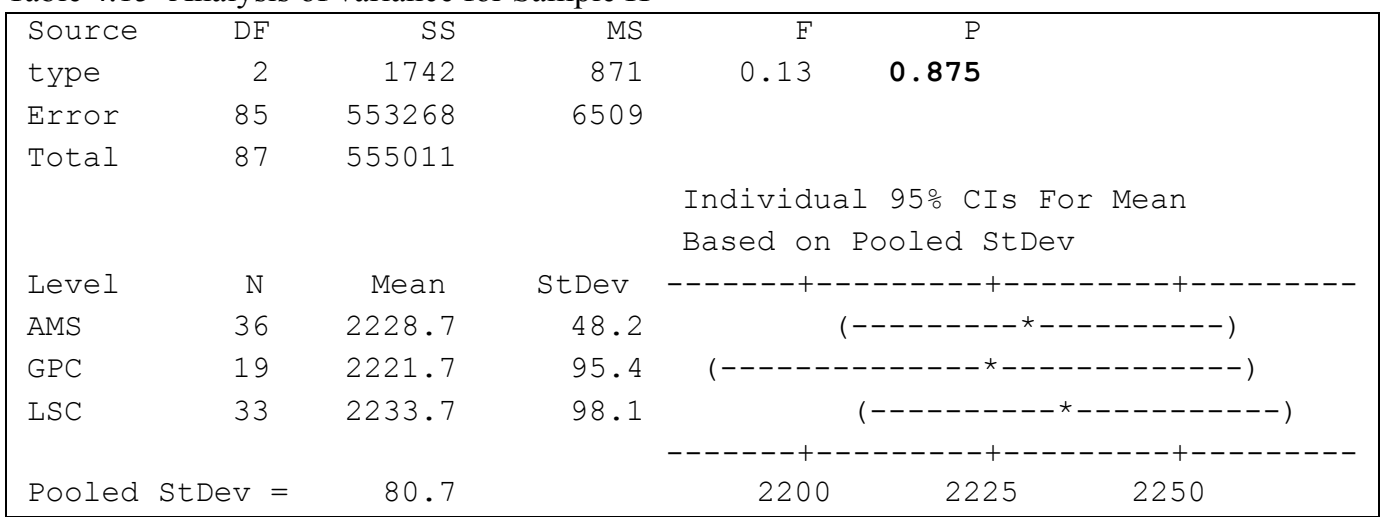

\subsubsection{Conclusion}

Since the p-value is $>0.05$ in Table 4.15 , there is no evidence of statistically significant differences among laboratory types. The results from each laboratory type broadly overlap. 


\subsubsection{Sample I: Belfast Cellulose}

Table 4.16 Summary statistics of age for Sample I

\begin{tabular}{|c|c|c|c|c|c|}
\hline Type & $\mathrm{N}$ & Number omitted & Mean & Median & StDev \\
\hline AMS & 35 & 0 & 4499.1 & 4490.0 & 74.1 \\
\hline GPC & 16 & 3 & 4438.0 & 4450.0 & 68.6 \\
\hline LSC & 35 & 11 & 4508.4 & 4520.0 & 128.1 \\
\hline
\end{tabular}

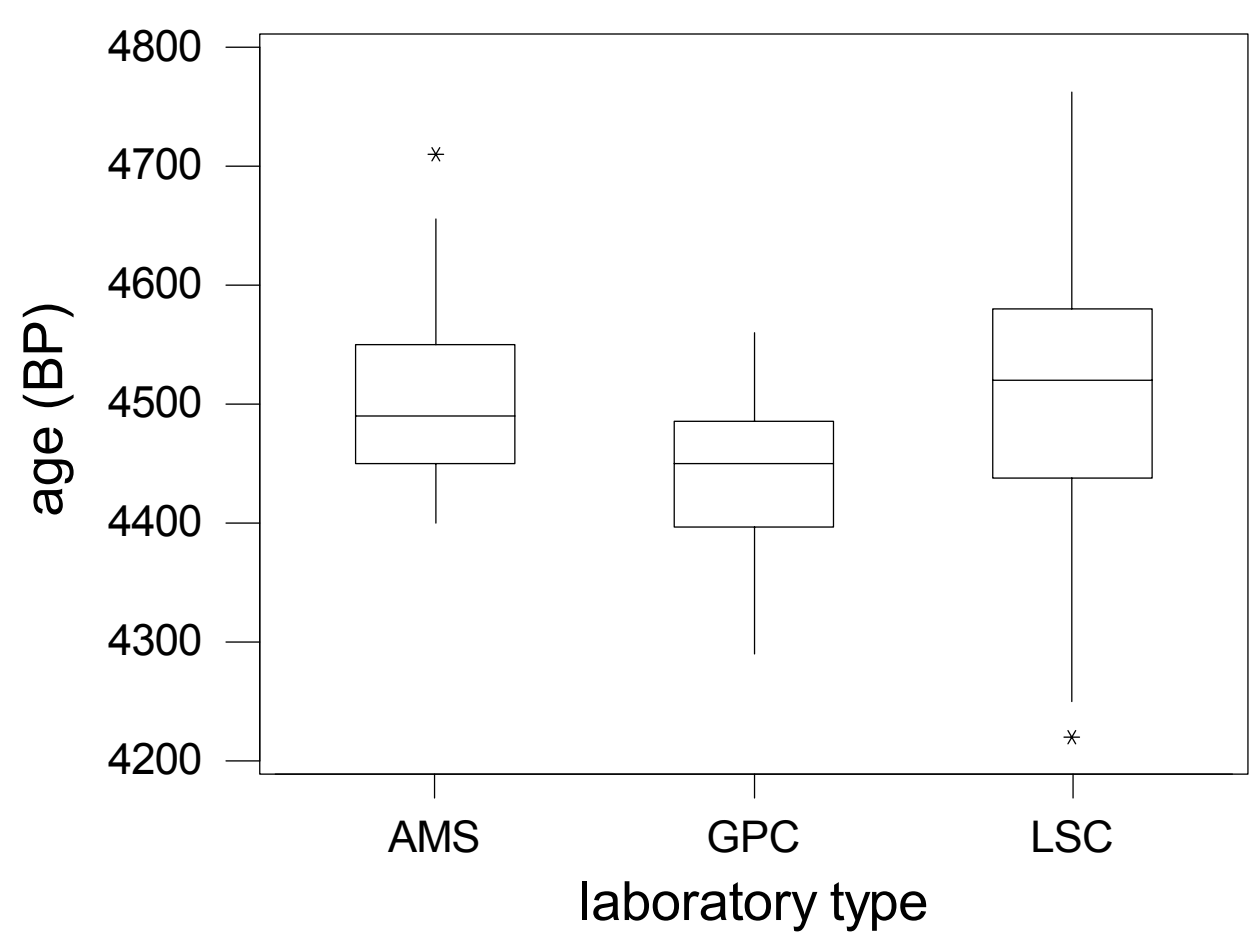

Figure 4.8 Distribution of age by laboratory type for Sample I

Table 4.17 Analysis of variance for Sample I

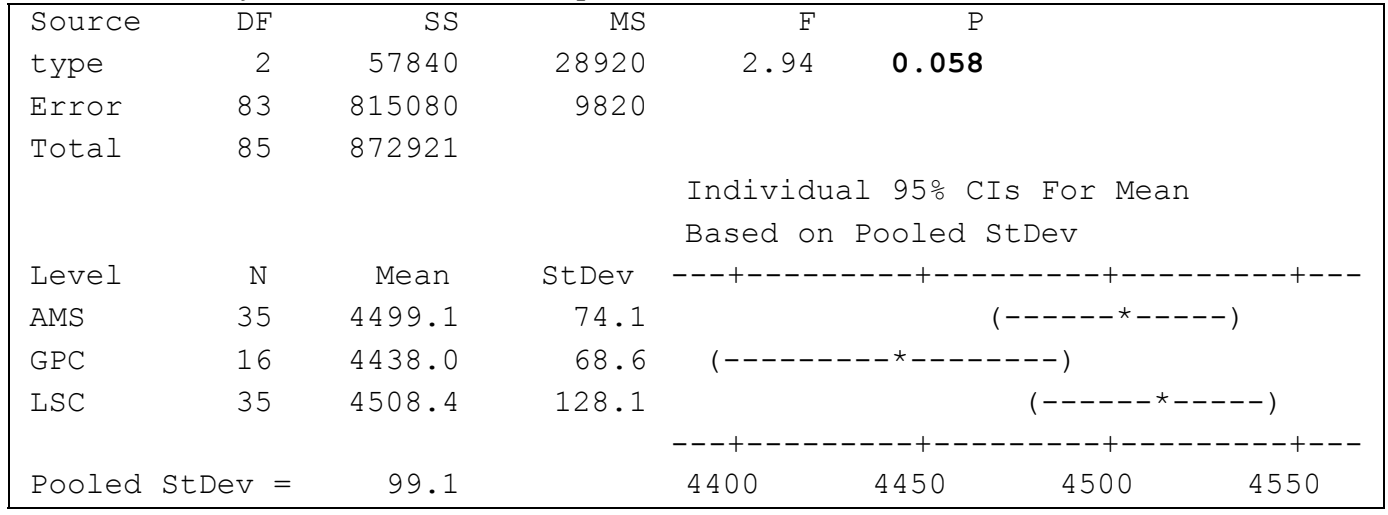




\subsubsection{Conclusion}

A statistically significant difference (at 10\%) is found among the laboratory types. GPC laboratories quote an average age that is lower than either AMS or LSC laboratories.

\subsubsection{Conclusions}

In the case of Samples F and I, a statistically significant difference among the laboratory types was found at a $10 \%$ level. In general for all other samples, there is no evidence of a difference, on average, among laboratory types. We can conclude that laboratories are comparable on the average age/activity. However, where we have seen lack of comparability is in the number of outliers, with LSC laboratories (as can be seen from the preceding tables) having by far the largest number of measurements omitted. The other striking feature from the tables is the comparison of the standard deviations, which provide a measure of the scatter or variation in the population. In all cases, the AMS laboratory results have the smallest variation, in some cases by as much as a factor of 2 .

\subsection{MODERN STANDARD AND BACKGROUND MATERIAL AS SOURCES OF VARIATION}

Two other factors of potential use in explaining the observed variation are the background and modern standard materials used. This section, thus, explores these 2 factors, with the reporting format identical to that used in Section 4.2. The classification of materials (and the analysis code used) is given below

Table 4.18 Coding for a) modern standard material and b) background material

a) Coding for modern standard material

\begin{tabular}{ll}
\hline Original description & Analysis classification/level \\
\hline ANU sucrose & ASuc \\
Benzene & Benz(ene) \\
NIST OxI & NBS1 \\
NIST OxII & NBS2 \\
GIN/HD-95,C-3 & Other \\
NIST 1/II & NBS12
\end{tabular}

b) Coding for background material

\begin{tabular}{ll}
\hline Original description & Analysis classification \\
\hline Anthracite & Anth(racite) \\
Benzene & Benz(ene) \\
Calcite & Calc(ite) \\
Charcoal & Charc(oal) \\
Bituminous coal & Coal \\
Graphite & Graphite \\
Doublespar/IAEA C1 & Marble \\
IAEA C4/wood/limestone & Other
\end{tabular}




\subsubsection{Sample C: Marine Turbidite}

Table 4.19a Descriptive statistics for age by modern standard used

\begin{tabular}{lrllll}
\hline Standard & N & Number omitted & Mean & Median & StDev \\
\hline ASuc & 8 & 1 & 18,294 & 18,225 & 217 \\
Benz & 3 & 2 & 17,918 & 17,820 & 211 \\
NBS1 & 25 & 5 & 18,143 & 18,180 & 215 \\
NBS12 & 9 & 0 & 18,123 & 18,100 & 188 \\
NBS2 & 27 & 2 & 18,149 & 18,120 & 231 \\
Other & 5 & 0 & 18,107 & 18,138 & 248 \\
Unknown & 7 & 1 & 18,115 & 18,140 & 135 \\
\hline
\end{tabular}

Table 4.19b Descriptive statistics for age by background material used

\begin{tabular}{lrllll}
\hline Background & N & Number omitted & Mean & Median & StDev \\
\hline Anth & 12 & 0 & 18,118 & 18,191 & 304 \\
Benz & 14 & 3 & 18,070 & 18,120 & 205 \\
Calc & 3 & 0 & 18,347 & 18,230 & 229 \\
Coal & 4 & 0 & 18,095 & 18,144 & 139 \\
Graph & 2 & 1 & 18,240 & 18,240 & 189 \\
Marble & 22 & 3 & 18,162 & 18,145 & 207 \\
Other & 14 & 3 & 18,157 & 18,227 & 216 \\
Unknown & 13 & 1 & 18,163 & 18,160 & 178 \\
\hline
\end{tabular}

\subsubsection{Graphical Analysis}

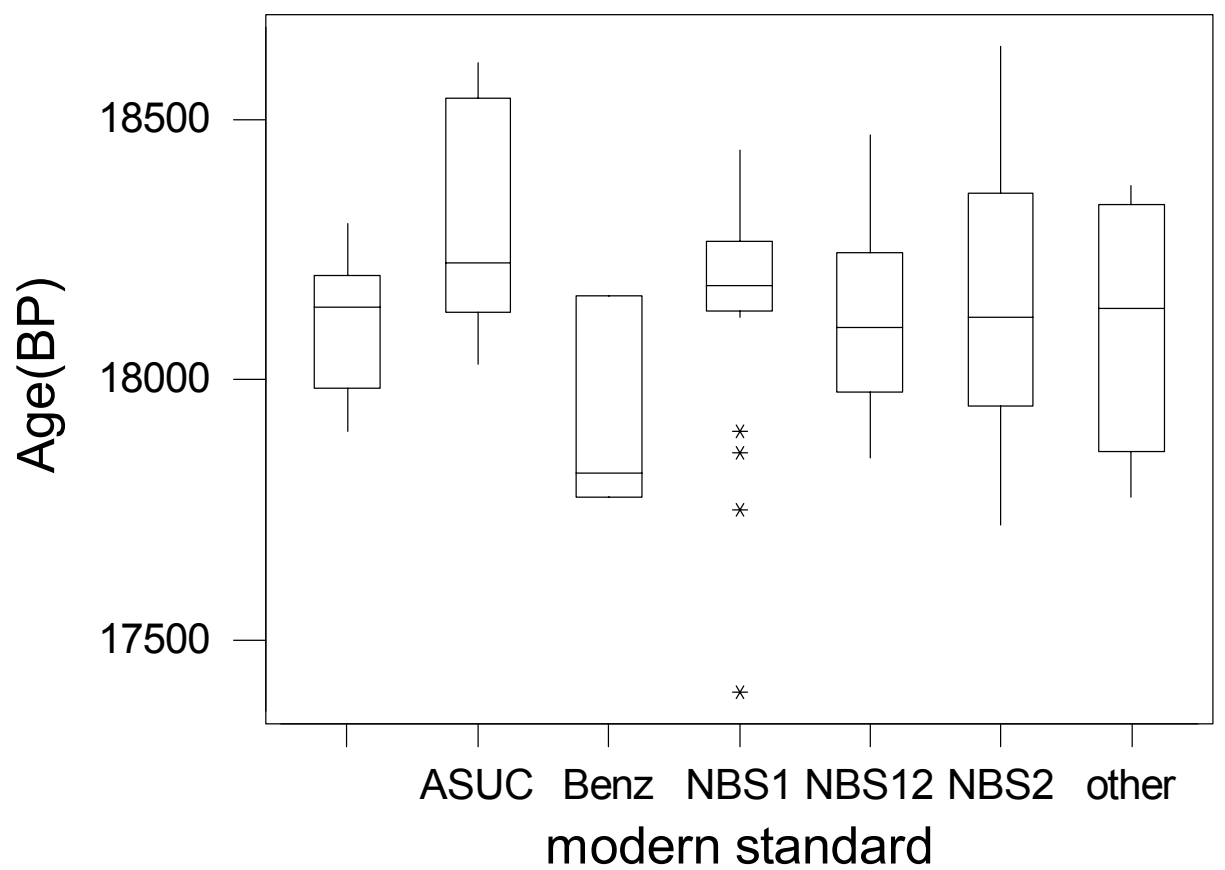

Figure 4.9a Distribution of age (yr BP) by modern standard material 


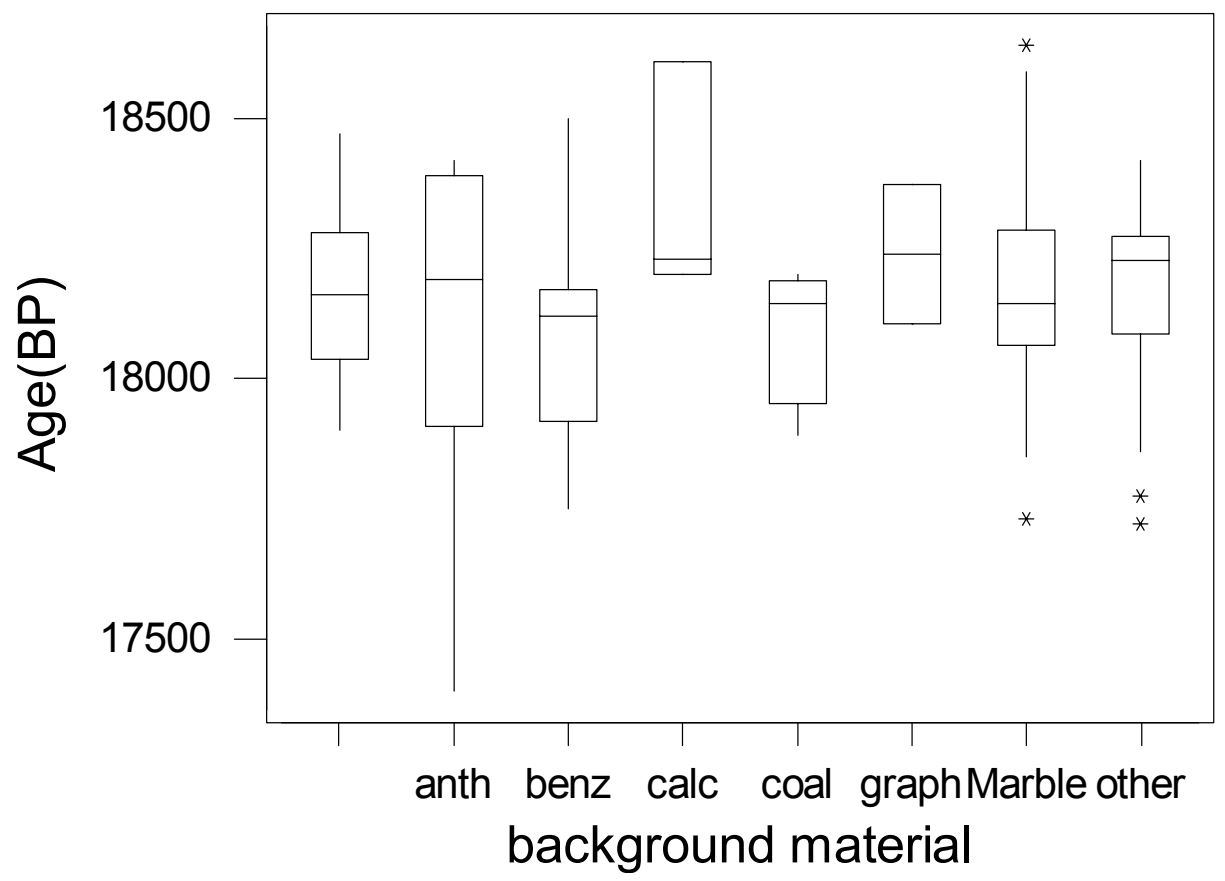

Figure 4.9b Distribution of age by background material

\subsubsection{Formal Analysis}

The formal analysis is carried out using a one-way analysis of variance (ANOVA); the hypothesis of interest is that the mean ${ }^{14} \mathrm{C}$ age is the same, irrespective of the modern standard or background material. The results are again summarized by the p-value.

Table 4.20a Analysis of variance of age by modern standard

\begin{tabular}{|c|c|c|c|c|}
\hline Source & $\mathrm{DF}$ & SS & MS & F \\
\hline Age & 5 & 342707 & 68541 & 0.230 \\
\hline Error & 71 & 3442176 & 48481 & \\
\hline \multirow[t]{2}{*}{ Total } & 76 & 3784883 & & \\
\hline & & & & $\begin{array}{l}\text { Individual 95\% CIs For Mean } \\
\text { Based on Pooled StDev }\end{array}$ \\
\hline Level & $\mathrm{N}$ & Mean & StDev & ----+----------+----------+----------+-- \\
\hline ASUC & 8 & 18294 & 217 & $(-----\star-----)$ \\
\hline Benz & 3 & 17918 & 211 & $(---------\star---------)$ \\
\hline NBS 1 & 25 & 18143 & 215 & $(---\star--)$ \\
\hline NBS12 & 9 & 18123 & 188 & $(-----*-----)$ \\
\hline NBS 2 & 27 & 18149 & 231 & $(--\star--)$ \\
\hline \multirow[t]{2}{*}{ other } & 5 & 18107 & 248 & $(-------\star-------)$ \\
\hline & & & & -----+----------+----------++---------++-- \\
\hline Pooled & StDev $=$ & 220 & & 18250 \\
\hline
\end{tabular}


Table 4.20b Analysis of variance of age by background material

\begin{tabular}{|c|c|c|c|c|}
\hline Source & $\mathrm{DF}$ & SS & MS & F \\
\hline Age & 6 & 244377 & 40729 & 0.576 \\
\hline Error & 64 & 3271008 & 51109 & \\
\hline \multirow{2}{*}{ Total } & 70 & 3515384 & & \\
\hline & & & & $\begin{array}{l}\text { Individual 95\% CIs For Mean } \\
\text { Based on Pooled StDev }\end{array}$ \\
\hline Level & $\mathrm{N}$ & Mean & StDev & -------+---------+---------+--------- \\
\hline anth & 12 & 18118 & 304 & $(------\star-----)$ \\
\hline benz & 14 & 18070 & 205 & $(------\star-----)$ \\
\hline calc & 3 & 18347 & 229 & $\left(------------{ }^{*}-------------\right)$ \\
\hline coal & 4 & 18095 & 139 & $(------------\star-----------)$ \\
\hline graph & 2 & 18240 & 189 & $(----------------\star---------------)$ \\
\hline Marble & 22 & 18162 & 207 & $(----\star----)$ \\
\hline other & 14 & 18157 & 216 & $(-----\star-----)$ \\
\hline Pooled & $\mathrm{ev}=$ & 226 & & 18200 \\
\hline
\end{tabular}

\subsubsection{Conclusion}

For Sample C, there is no evidence that either the modern standard or background material used is a statistically significant factor in explaining the variation observed ( $p$-value $>0.05$ in both cases).

\subsubsection{Sample D: Belfast Wood}

Table 4.21a Descriptive statistics age by modern standard material

\begin{tabular}{lrlllc}
\hline Standard & N & Number omitted & Mean & Median & StDev \\
\hline ASUC & 6 & 1 & 4558.5 & 4565.0 & 95.4 \\
Benz & 3 & 3 & 4416.7 & 4420.0 & 90.0 \\
NBS1 & 28 & 3 & 4505.4 & 4510.5 & 52.9 \\
NBS12 & 7 & 0 & 4567.7 & 4550.0 & 51.4 \\
NBS2 & 37 & 3 & 4537.8 & 4540.0 & 82.6 \\
Other & 7 & 1 & 4485.4 & 4482.0 & 35.3 \\
Unknown & 10 & 2 & 4516.8 & 4517.0 & 108.1 \\
\hline
\end{tabular}

Table 4.21b Descriptive statistics age by background material

\begin{tabular}{lrlllr}
\hline Background & N & Number omitted & Mean & Median & StDev \\
\hline Anth & 19 & 1 & 4530.3 & 4511.0 & 74.2 \\
Benz & 14 & 4 & 4515.1 & 4502.5 & 124.6 \\
Calc & 2 & 0 & 4530.0 & 4530.0 & 28.3 \\
Charc & 2 & 0 & 4525.0 & 4525.0 & 134.4 \\
Coal & 11 & 0 & 4487.9 & 4510.0 & 55.9 \\
Graph & 9 & 0 & 4513.7 & 4500.0 & 41.9 \\
Marble & 6 & 2 & 4505.5 & 4527.5 & 102.9 \\
Other & 18 & 3 & 4521.7 & 4519.0 & 56.6 \\
Unknown & 17 & 3 & 4551.8 & 4540.0 & 74.6 \\
\hline
\end{tabular}




\subsubsection{Graphical Analysis}

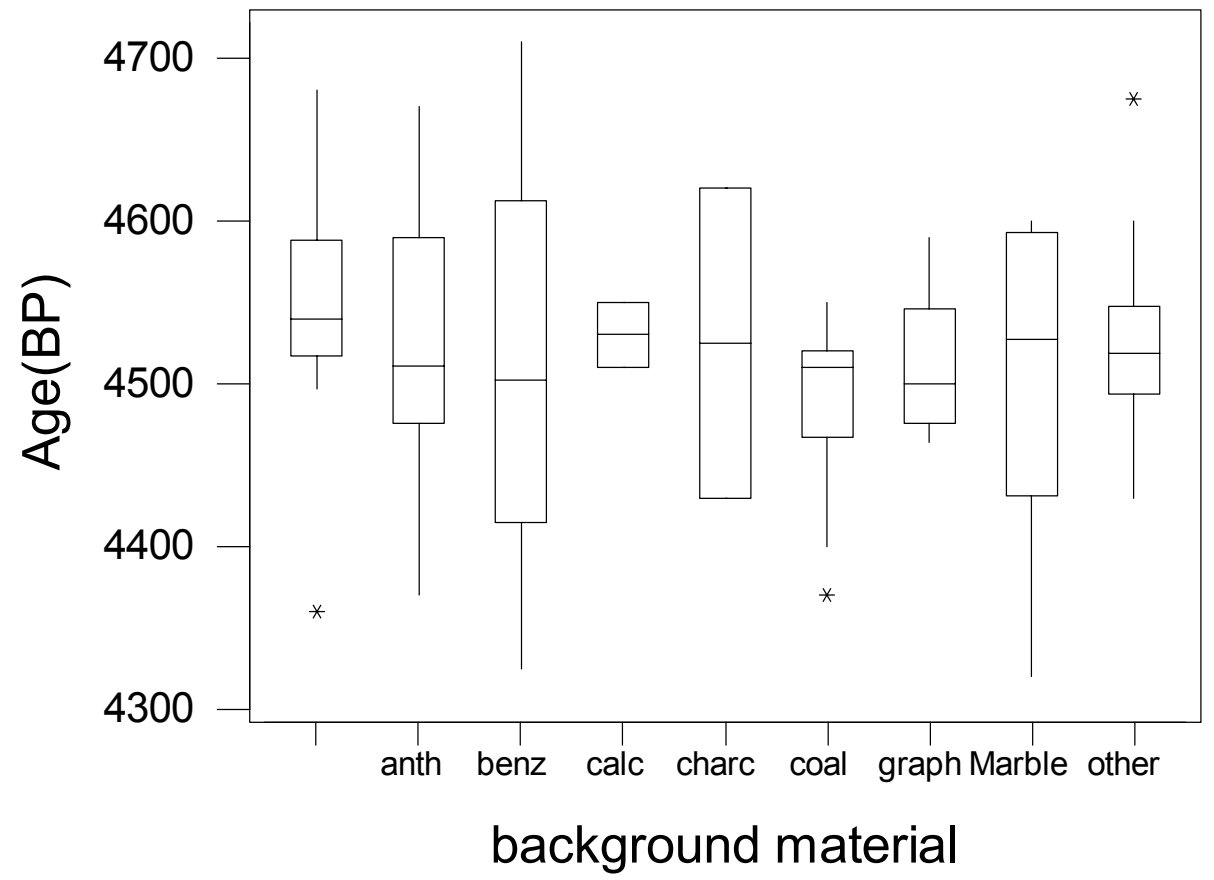

Figure 4.10a Distribution of age by background material

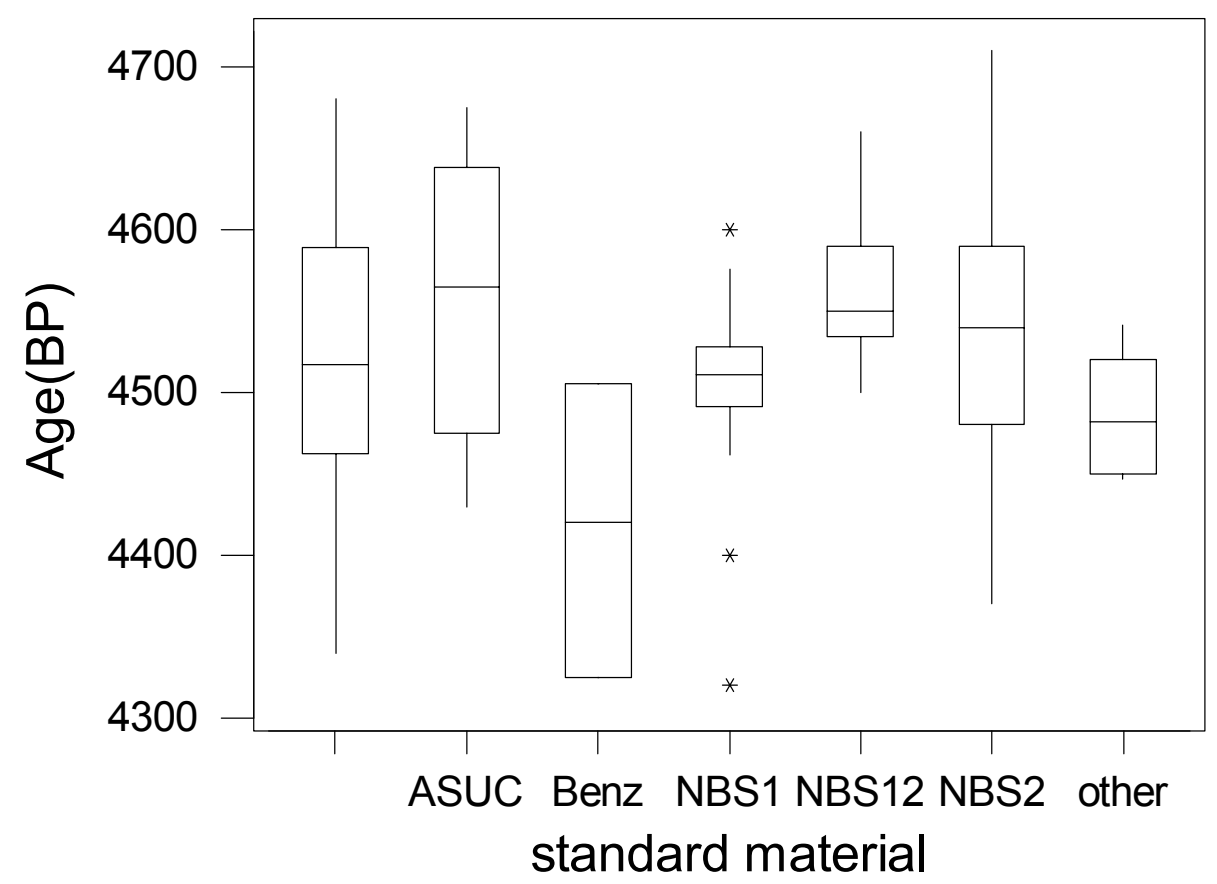

Figure 4.10b Distribution of age by modern standard material 


\subsubsection{Formal Analysis}

Table 4.22a Analysis of variance of age by modern standard material

\begin{tabular}{|c|c|c|c|c|}
\hline Source & $\mathrm{DF}$ & SS & MS & $\mathrm{F}$ \\
\hline age & 5 & 82173 & 16435 & 0.009 \\
\hline Error & 82 & 406320 & 4955 & \\
\hline \multirow[t]{2}{*}{ Total } & 87 & 488493 & & \\
\hline & & & & $\begin{array}{l}\text { Individual 95\% CIs For Mean } \\
\text { Based on Pooled StDev }\end{array}$ \\
\hline Level & $\mathrm{N}$ & Mean & StDev & ---------+---------+---------+------- \\
\hline ASUC & 6 & 4558.5 & 95.4 & $(------\star------)$ \\
\hline Benz & 3 & 4416.7 & 90.0 & $(---------\star---------)$ \\
\hline NBS1 & 28 & 4505.4 & 52.9 & $(--\star--)$ \\
\hline NBS12 & 7 & 4567.7 & 51.4 & $(------\star------)$ \\
\hline NBS2 & 37 & 4537.8 & 82.6 & $(--\star--)$ \\
\hline other & 7 & 4485.4 & 35.3 & $(------\star-----)$ \\
\hline Pooled & StDev $=$ & 70.4 & & 4560 \\
\hline
\end{tabular}

Table $4.22 \mathrm{~b}$ Analysis of variance of age by background material

\begin{tabular}{|c|c|c|c|c|}
\hline Source & $\overline{\mathrm{DF}}$ & SS & MS & $\mathrm{F}$ \\
\hline age & 7 & 14421 & 2060 & 0.944 \\
\hline Error & 73 & 472634 & 6474 & \\
\hline \multirow[t]{2}{*}{ Total } & 80 & 487055 & & \\
\hline & & & & $\begin{array}{l}\text { Individual 95\% CIs For Mean } \\
\text { Based on Pooled StDev }\end{array}$ \\
\hline Level & $\mathrm{N}$ & Mean & StDev & ----------+---------+---------+------ \\
\hline anth & 19 & 4530.3 & 74.2 & $(----\star----)$ \\
\hline benz & 14 & 4515.1 & 124.6 & $(-----\star-----)$ \\
\hline $\mathrm{calc}$ & 2 & 4530.0 & 28.3 & $(---------------*---------------)$ \\
\hline charc & 2 & 4525.0 & 134.4 & $(---------------\star----------------)$ \\
\hline coal & 11 & 4487.9 & 55.9 & $(------\star------)$ \\
\hline graph & 9 & 4513.7 & 41.9 & $(-------\star------)$ \\
\hline Marble & 6 & 4505.5 & 102.9 & $(---------\star--------)$ \\
\hline other & 18 & 4521.7 & 56.6 & $(----\star----)$ \\
\hline Pooled & StDev $=$ & 80.5 & & 4620 \\
\hline
\end{tabular}

\subsubsection{Conclusions}

For Sample D, the modern standard is found to be statistically significant in explaining the observed variation. There are statistically significant differences among the mean ages for results based on the different modern standards. The modern standard material as a factor accounts for approximately $17 \%$ of the total variation observed. Laboratories using benzene as a modern standard material quote, on average, lower ages for this sample. The background material was not found statistically significant. 


\subsubsection{Sample F: Belfast Wood}

Table 4.23a Descriptive statistics: age by background material

\begin{tabular}{lrllll}
\hline Background & N & Number omitted & Mean & Median & StDev \\
\hline Anth & 16 & 3 & 4507.9 & 4502.0 & 83.0 \\
Benz & 14 & 4 & 4507.1 & 4495.0 & 82.4 \\
Calc & 2 & 0 & 4472.5 & 4472.5 & 46.0 \\
Charc & 2 & 0 & 4525.0 & 4525.0 & 91.9 \\
Coal & 8 & 1 & 4461.8 & 4477.0 & 71.4 \\
Graph & 10 & 1 & 4554.9 & 4555.0 & 73.8 \\
Marble & 6 & 2 & 4551.8 & 4550.0 & 98.9 \\
Other & 18 & 2 & 4519.1 & 4511.5 & 66.6 \\
Unknown & 14 & 3 & 4488.9 & 4491.5 & 69.4 \\
\hline
\end{tabular}

Table 4.23b Descriptive statistics: age by modern standard

\begin{tabular}{lrlllr}
\hline Standard & N & Number omitted & Mean & Median & StDev \\
\hline Asuc & 5 & 3 & 4546.0 & 4550.0 & 63.5 \\
Benz & 5 & 1 & 4470.2 & 4459.0 & 116.0 \\
NBS1 & 28 & 3 & 4502.4 & 4497.0 & 48.0 \\
NBS12 & 9 & 0 & 4566.3 & 4550.0 & 63.8 \\
NBS2 & 29 & 6 & 4518.4 & 4513.0 & 97.4 \\
Other & 8 & 0 & 4503.3 & 4487.0 & 42.1 \\
Unknown & 6 & 3 & 4442.8 & 4460.0 & 59.4 \\
\hline
\end{tabular}

\subsubsection{Graphical Analysis}

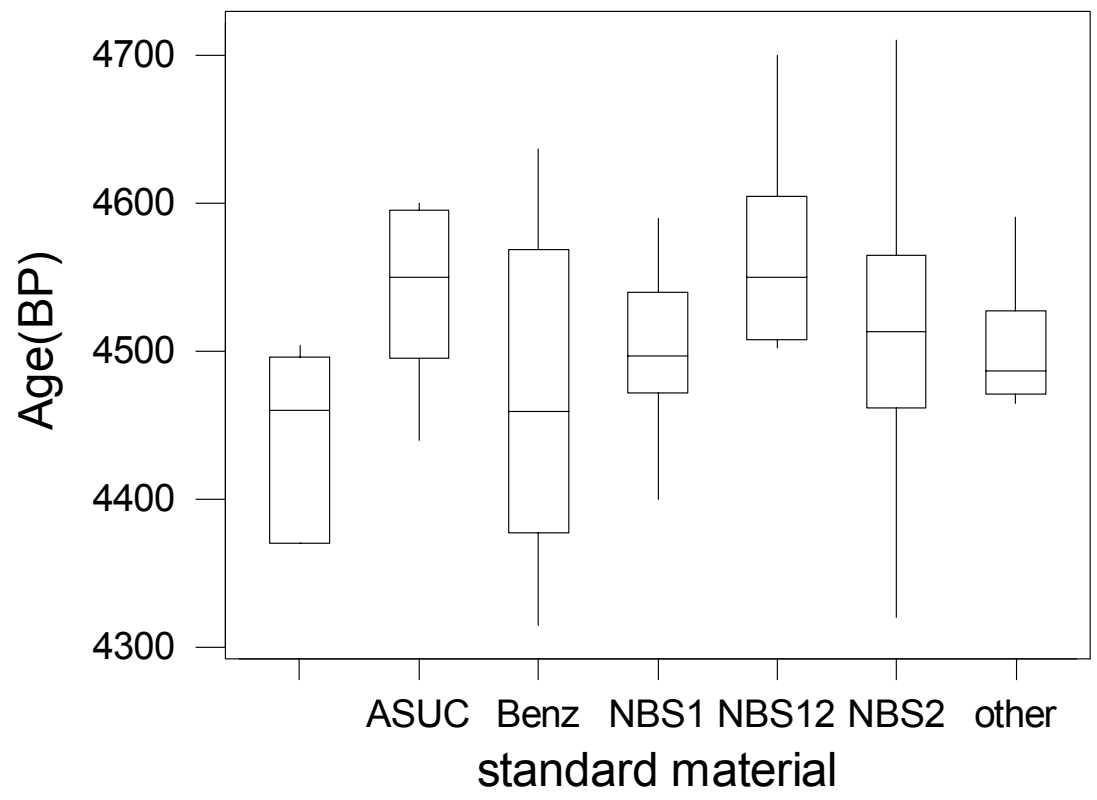

Figure 4.11a Distribution of age by modern standard material 


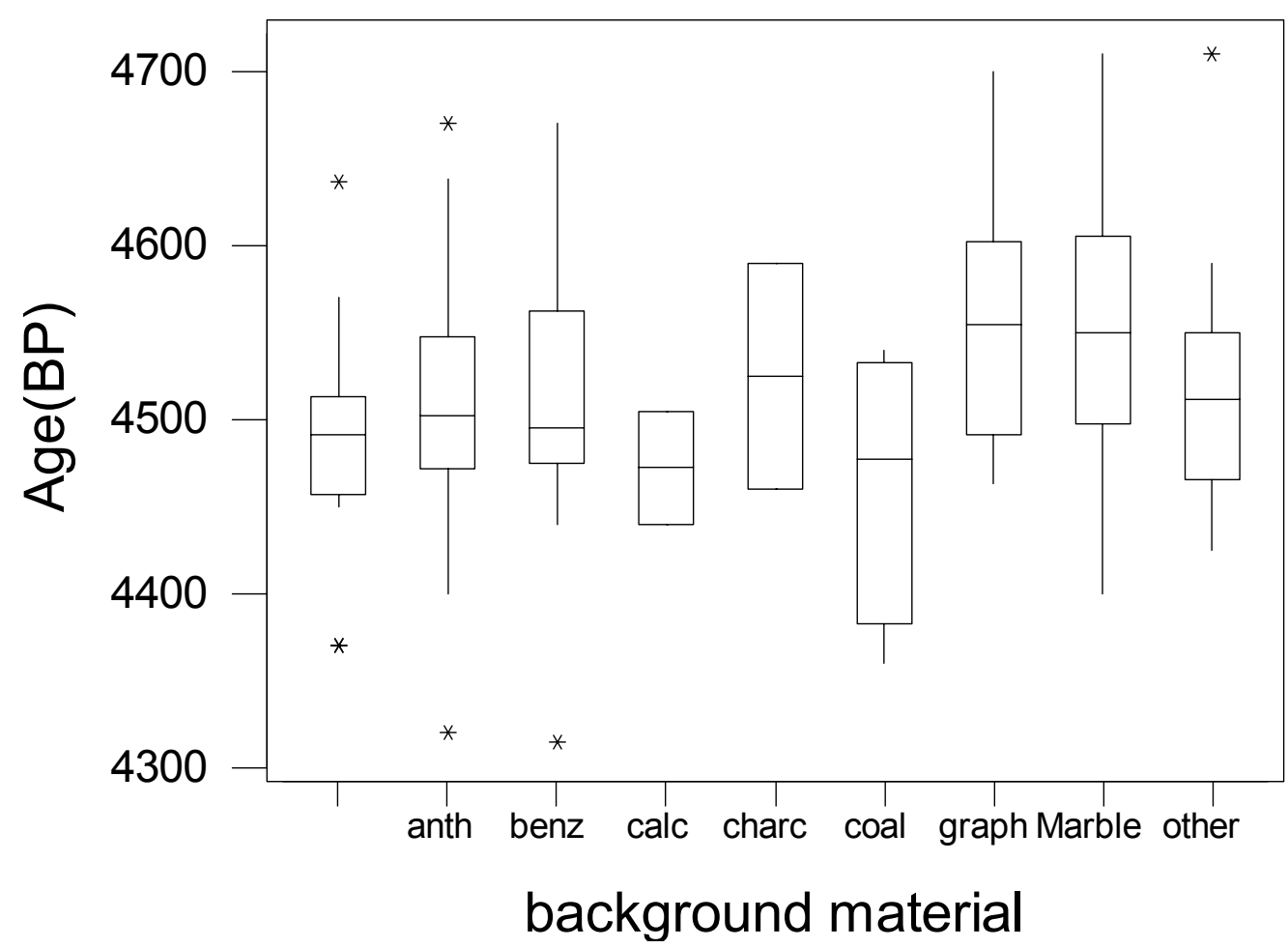

Figure 4.11b Distribution of age by background material

\subsubsection{Formal Analysis}

Table 4.24a Analysis of variance of age by background

\begin{tabular}{|c|c|c|c|c|}
\hline Source & $\mathrm{DF}$ & SS & MS & F \\
\hline age & 7 & 52484 & 7498 & 0.294 \\
\hline Error & 68 & 411176 & 6047 & \\
\hline \multirow[t]{2}{*}{ Total } & 75 & 463660 & & \\
\hline & & & & $\begin{array}{l}\text { Individual 95\% CIs For Mean } \\
\text { Based on Pooled StDev }\end{array}$ \\
\hline Level & $\mathrm{N}$ & Mean & StDev & -----+---------+---------+---------+- \\
\hline anth & 16 & 4507.9 & 83.0 & $(---\star----)$ \\
\hline benz & 14 & 4507.1 & 82.4 & $(----\star-----)$ \\
\hline calc & 2 & 4472.5 & 46.0 & $(-------------\star-------------)$ \\
\hline charc & 2 & 4525.0 & 91.9 & $(-------------\star------------)$ \\
\hline coal & 8 & 4461.8 & 71.4 & $(------\star------)$ \\
\hline graph & 10 & 4554.9 & 73.8 & $(-----\star-----)$ \\
\hline Marble & 6 & 4551.8 & 98.9 & $(-------\star-------)$ \\
\hline other & 18 & 4519.1 & 66.6 & $(----\star---)$ \\
\hline Pooled & StDev $=$ & 77.8 & & 4560 \\
\hline
\end{tabular}


Table 4.24b Analysis of variance of age by modern standard

\begin{tabular}{|c|c|c|c|c|}
\hline Source & $\mathrm{DF}$ & SS & MS & F \\
\hline Age & 5 & 44382 & 8876 & 0.180 \\
\hline Error & 78 & 442573 & 5674 & \\
\hline \multirow[t]{2}{*}{ Total } & 83 & 486955 & & \\
\hline & & & & $\begin{array}{l}\text { Individual 95\% CIs For Mean } \\
\text { Based on Pooled StDev }\end{array}$ \\
\hline Level & $\mathrm{N}$ & Mean & StDev & --------+----------+---------++--------- \\
\hline ASUC & 5 & 4546.0 & 63.5 & $(-----------\star *----------)$ \\
\hline Benz & 5 & 4470.2 & 116.0 & $(----------*----------)$ \\
\hline NBS 1 & 28 & 4502.4 & 48.0 & $(---\star----)$ \\
\hline NBS 12 & 9 & 4566.3 & 63.8 & $(-------\star-------)$ \\
\hline NBS 2 & 29 & 4518.4 & 97.4 & $(----\star----)$ \\
\hline other & 8 & 4503.3 & 42.1 & $(--------\star-------)$ \\
\hline Pooled & StDev $=$ & 75.3 & & 4560 \\
\hline
\end{tabular}

\subsubsection{Conclusions}

Neither modern standard nor background materials proved to be statistically significant in explaining the observed variation.

\subsubsection{Sample E: Humic Acid}

Table 4.25a Descriptive statistics: age by modern standard

\begin{tabular}{lrlllr}
\hline Standard & N & Number omitted & Mean & Median & StDev \\
\hline ASUC & 8 & 2 & 11,712 & 11,715 & 130 \\
Benz & 4 & 2 & 11,681 & 11,682 & 58 \\
NBS1 & 49 & 7 & 11,779 & 11,800 & 144 \\
NBS12 & 5 & 0 & 11,781 & 11,770 & 87 \\
NBS2 & 37 & 6 & 11,813 & 11,809 & 167 \\
Other & 14 & 0 & 11,785 & 11,771 & 175 \\
Unknown & 8 & 2 & 11,710 & 11,721 & 91 \\
\hline
\end{tabular}

Table 4.25 b Descriptive statistics: age by background material

\begin{tabular}{lrlllr}
\hline Background & N & Number omitted & Mean & Median & StDev \\
\hline Anth & 20 & 3 & 11,847 & 11,855 & 139 \\
Benz & 18 & 4 & 11,761 & 11,700 & 208 \\
Calc & 2 & 0 & 11,715 & 11,715 & 78 \\
Charc & 13 & 0 & 11,832 & 11,800 & 90 \\
Coal & 18 & 0 & 11,804 & 11,805 & 141 \\
Graph & 15 & 0 & 11,734 & 11,772 & 158 \\
Marble & 9 & 2 & 11,754 & 11,760 & 176 \\
Other & 17 & 5 & 11,729 & 11,731 & 126 \\
Unknown & 13 & 5 & 11,748 & 11,760 & 103 \\
\hline
\end{tabular}




\subsubsection{Graphical Analysis}

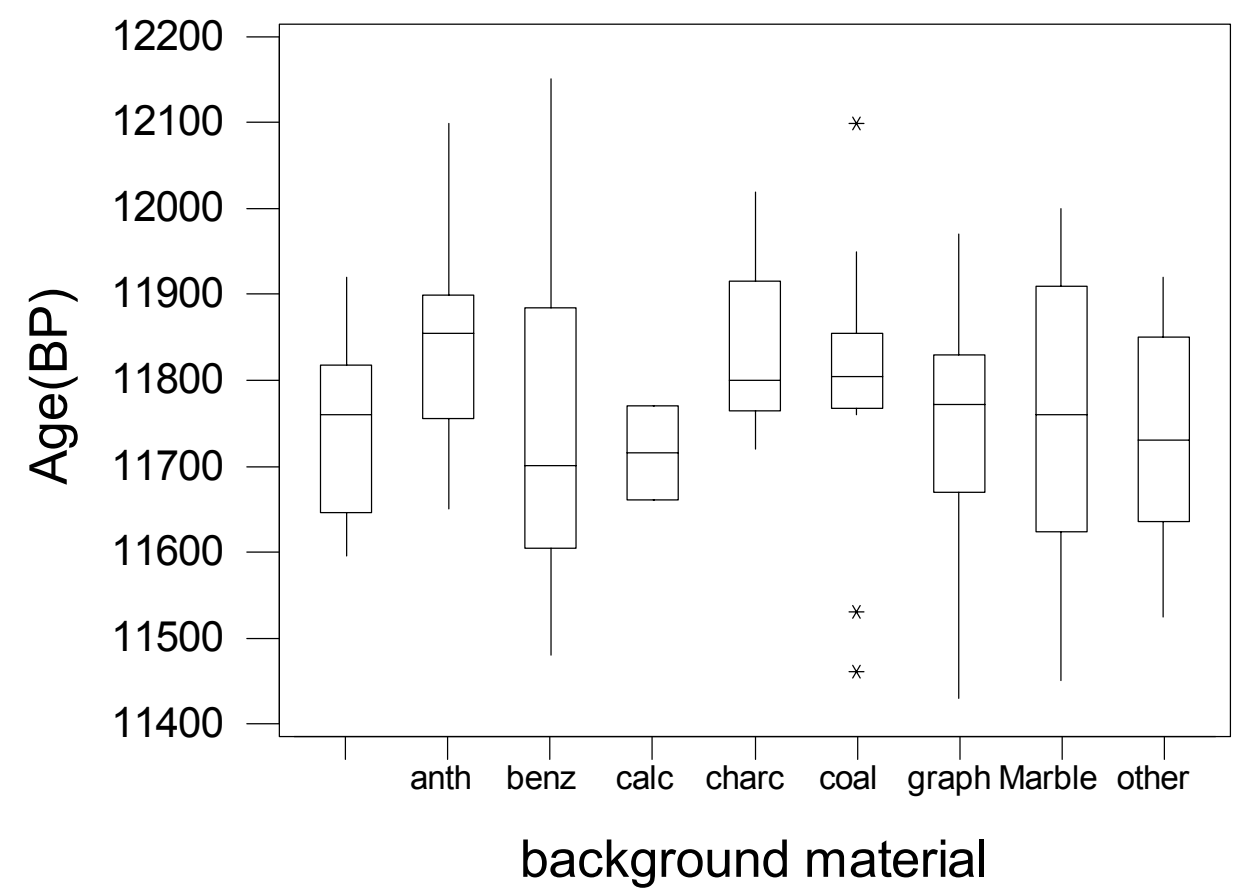

Figure 4.12a Distribution of age by background material

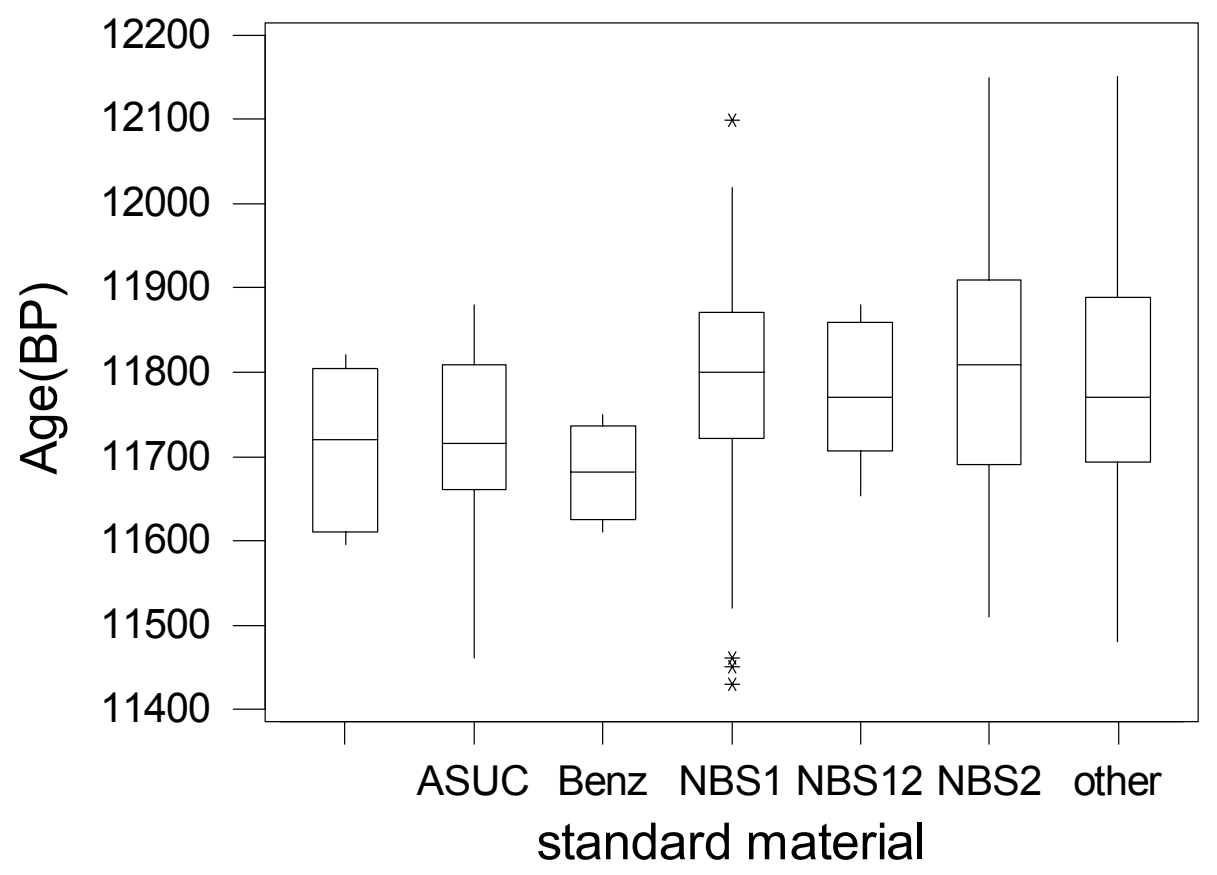

Figure 4.12b Distribution of age by modern standard material 


\subsubsection{Formal Analysis}

Table 4.26a Analysis of variance of age by modern standard

\begin{tabular}{|c|c|c|c|c|}
\hline Source & $\mathrm{DF}$ & SS & MS & F \\
\hline Age & 5 & 117339 & 23468 & 0.409 \\
\hline Error & 111 & 2552299 & 22994 & \\
\hline \multirow[t]{2}{*}{ Total } & 116 & 2669638 & & \\
\hline & & & & $\begin{array}{l}\text { Individual 95\% CIs For Mean } \\
\text { Based on Pooled StDev }\end{array}$ \\
\hline Level & $\mathrm{N}$ & Mean & StDev & -----------+----------+----------++------ \\
\hline ASUC & 8 & 11712 & 130 & $(--------\star--------)$ \\
\hline Benz & 4 & 11681 & 58 & $(-----------* \star------------)$ \\
\hline NBS 1 & 49 & 11779 & 144 & $(---\star--)$ \\
\hline NBS 12 & 5 & 11781 & 87 & $(----------\star----------)$ \\
\hline NBS2 & 37 & 11813 & 167 & $(---\star----)$ \\
\hline other & 14 & 11785 & 175 & $(------\star------)$ \\
\hline Pooled & StDev $=$ & 152 & & $\begin{array}{lll}11640 & 11760 & 11880\end{array}$ \\
\hline
\end{tabular}

Table 4.26b Analysis of variance of age by background material

\begin{tabular}{|c|c|c|c|c|}
\hline Source & $\mathrm{DF}$ & SS & $\mathrm{MS}$ & F \\
\hline backgroun & 7 & 234848 & 33550 & 0.190 \\
\hline Error & 104 & 2392794 & 23008 & \\
\hline \multirow[t]{2}{*}{ Total } & 111 & 2627641 & & \\
\hline & & & & $\begin{array}{l}\text { Individual 95\% CIs For Mean } \\
\text { Based on Pooled StDev }\end{array}$ \\
\hline Level & $\mathrm{N}$ & Mean & StDev & --+---------+----------+---------+---- \\
\hline anth & 20 & 11847 & 139 & $(----\star-----)$ \\
\hline benz & 18 & 11761 & 208 & $(-----\star-----)$ \\
\hline calc & 2 & 11715 & 78 & $(----------------\star-----------------)$ \\
\hline charc & 13 & 11832 & 90 & $(------\star------)$ \\
\hline coal & 18 & 11804 & 141 & $(-----\star-----)$ \\
\hline graph & 15 & 11734 & 158 & $(------\star-----)$ \\
\hline Marble & 9 & 11754 & 176 & $(-------\star--------)$ \\
\hline other & 17 & 11729 & 126 & $(-----\star------)$ \\
\hline Pooled StI & ev $=$ & 152 & & 11880 \\
\hline
\end{tabular}

\subsubsection{Conclusions}

For Sample E, neither modern standard nor background materials are statistically significant in explaining the observed variation. 


\subsubsection{Sample G: Barley Mash}

Table 4.27a Descriptive statistics: activity (pMC) by background material

\begin{tabular}{lrllll}
\hline Background & $\mathrm{N}$ & Number omitted & Mean & Median & StDev \\
\hline Anth & 15 & 3 & 110.30 & 110.86 & 1.48 \\
Benz & 16 & 3 & 110.46 & 110.85 & 1.28 \\
Calc & 2 & 0 & 110.27 & 110.27 & 0.01 \\
Charc & 2 & 0 & 110.90 & 110.90 & 0.70 \\
Coal & 7 & 0 & 110.14 & 110.13 & 1.70 \\
Graph & 10 & 0 & 110.33 & 110.49 & 0.64 \\
Marble & 6 & 2 & 110.83 & 110.75 & 0.99 \\
Other & 16 & 3 & 110.35 & 110.28 & 0.81 \\
Unknown & 12 & 3 & 110.71 & 110.80 & 0.66 \\
\hline
\end{tabular}

Table 4.27b Descriptive statistics: activity (pMC) by modern standard

\begin{tabular}{lrllll}
\hline Standard & N & Number omitted & Mean & Median & StDev \\
\hline ASUC & 6 & 1 & 109.39 & 109.75 & 1.32 \\
Benz & 5 & 1 & 111.10 & 111.03 & 1.10 \\
NBS1 & 26 & 3 & 110.46 & 110.22 & 0.92 \\
NBS12 & 7 & 0 & 110.36 & 110.26 & 0.62 \\
NBS2 & 29 & 6 & 110.39 & 110.53 & 1.36 \\
Other & 7 & 1 & 110.82 & 110.84 & 0.40 \\
Unknown & 6 & 2 & 110.68 & 110.75 & 0.19 \\
\hline
\end{tabular}

\subsubsection{Graphical Analysis}

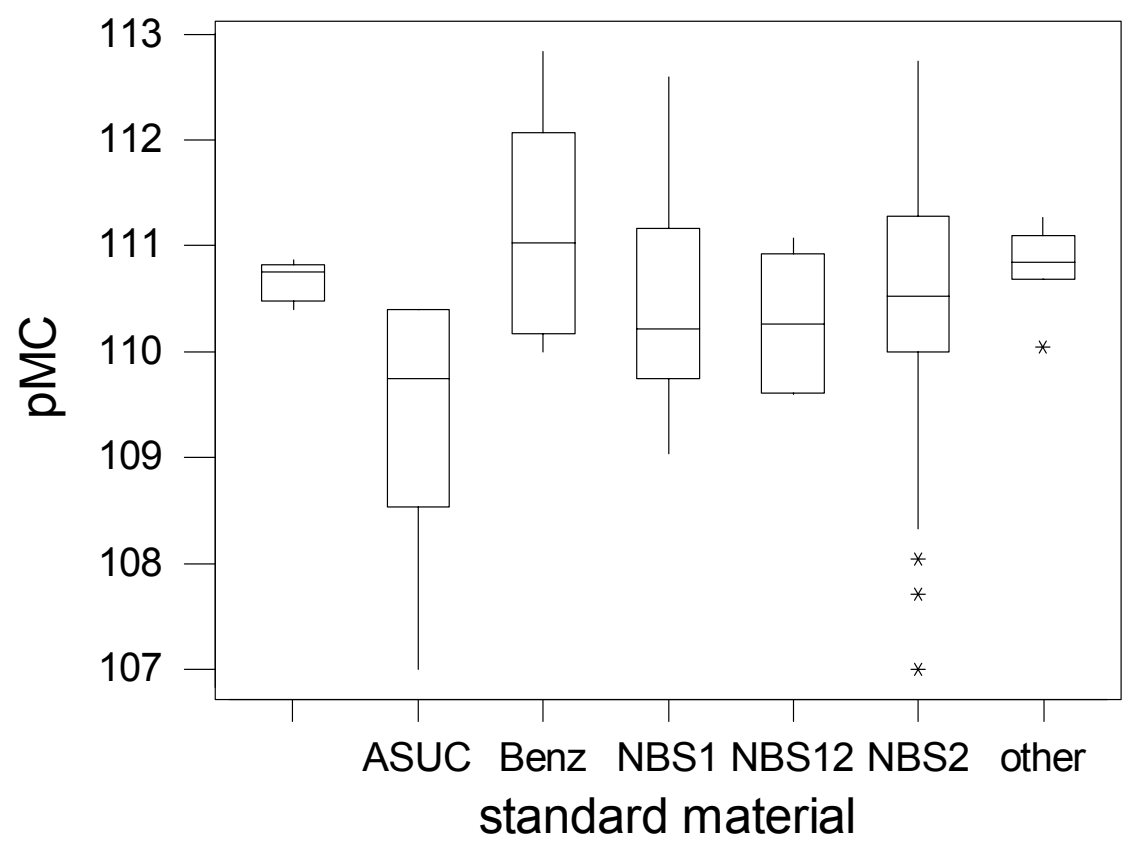

Figure 4.13a Distribution of activity by modern standard 


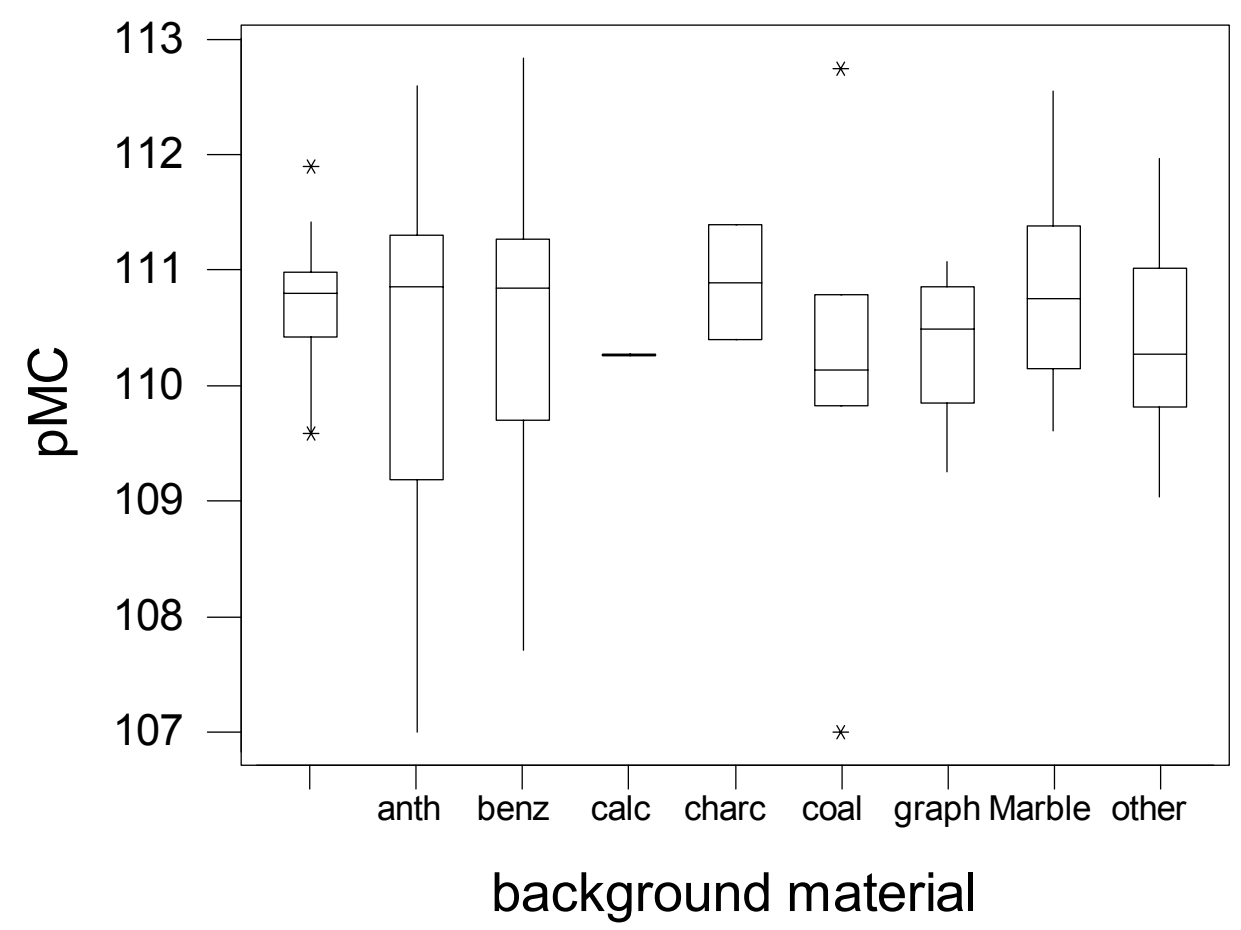

Figure 4.13b Distribution of activity by background material

\subsubsection{Formal Analysis}

Table 4.28a Analysis of variance of activity by background material

\begin{tabular}{|c|c|c|c|c|}
\hline Source & $\mathrm{DF}$ & SS & MS & $\mathrm{F}$ \\
\hline background & 7 & 2.42 & 0.35 & 0.971 \\
\hline Error & 66 & 91.67 & 1.39 & \\
\hline \multirow[t]{2}{*}{ Total } & 73 & 94.09 & & \\
\hline & & & & $\begin{array}{l}\text { Individual 95\% CIs For Mean } \\
\text { Based on Pooled StDev }\end{array}$ \\
\hline Level & $\mathrm{N}$ & Mean & StDev & ------+----------++----------+----------+- \\
\hline anth & 15 & 110.30 & 1.48 & $(----\star----)$ \\
\hline benz & 16 & 110.46 & 1.28 & $(----\star---)$ \\
\hline calc & 2 & 110.27 & 0.01 & $\left(-------------{ }^{*}-------------\right)$ \\
\hline charc & 2 & 110.90 & 0.70 & $\left(-------------{ }^{\star} \star-------------\right)$ \\
\hline coal & 7 & 110.14 & 1.70 & $(-------\star------)$ \\
\hline graph & 10 & 110.33 & 0.64 & $(-----\star------)$ \\
\hline Marble & 6 & 110.83 & 0.99 & $(-------\star-------)$ \\
\hline other & 16 & 110.35 & 0.81 & $(----\star---)$ \\
\hline Pooled StDe & $\mathrm{ev}=$ & 1.18 & & 111.6 \\
\hline
\end{tabular}


Table $4.28 \mathrm{~b}$ Analysis of variance of activity by modern standard

\begin{tabular}{|c|c|c|c|c|}
\hline Source & $\mathrm{DF}$ & SS & $\mathrm{MS}$ & F \\
\hline standard & 5 & 9.86 & 1.97 & 0.162 \\
\hline Error & 74 & 89.45 & 1.21 & \\
\hline Total & 79 & 99.31 & & $\begin{array}{l}\text { Individual 95\% CIs For Mean } \\
\text { Based on Pooled StDev }\end{array}$ \\
\hline Level & $\mathrm{N}$ & Mean & StDev & ------+---------+---------+---------+ \\
\hline ASUC & 6 & 109.39 & 1.32 & $(--------\star--------)$ \\
\hline Benz & 5 & 111.10 & 1.10 & $(---------\star---------)$ \\
\hline NBS 1 & 26 & 110.46 & 0.92 & $(----\star---)$ \\
\hline NBS 12 & 7 & 110.36 & 0.62 & $(--------\star-------)$ \\
\hline NBS 2 & 29 & 110.39 & 1.36 & $(---\star---)$ \\
\hline other & 7 & 110.82 & 0.40 & $(-------\star-------)$ \\
\hline Pooled St & $\mathrm{ev}=$ & 1.10 & & 111.0 \\
\hline
\end{tabular}

\subsubsection{Conclusions}

Neither modern standard nor background materials are statistically significant in explaining the variation in activity.

\subsubsection{Sample J: Barley Mash}

Table 4.29a Descriptive statistics: activity (pMC) by modern standard

\begin{tabular}{lrllll}
\hline Standard & N & Number omitted & Mean & Median & StDev \\
\hline ASUC & 4 & 1 & 109.89 & 109.83 & 1.19 \\
Benz & 5 & 1 & 110.63 & 110.22 & 1.30 \\
NBS1 & 27 & 2 & 110.52 & 110.50 & 0.77 \\
NBS12 & 8 & 0 & 110.77 & 110.56 & 0.78 \\
NBS2 & 28 & 7 & 110.64 & 110.71 & 0.98 \\
Other & 8 & 0 & 111.13 & 111.01 & 0.59 \\
Unknown & 7 & 2 & 110.40 & 110.90 & 1.12 \\
\hline
\end{tabular}

Table 4.29b Descriptive statistics: activity (pMC) by background material

\begin{tabular}{lrllll}
\hline Background & $\mathrm{N}$ & Number omitted & Mean & Median & StDev \\
\hline Anth & 17 & 1 & 110.45 & 110.70 & 1.15 \\
Benz & 13 & 5 & 111.23 & 111.03 & 0.80 \\
Calc & 2 & 0 & 110.00 & 110.00 & 0.24 \\
Charc & 2 & 0 & 111.39 & 111.39 & 0.01 \\
Coal & 7 & 0 & 110.51 & 110.92 & 0.81 \\
Graph & 10 & 1 & 110.85 & 110.61 & 0.64 \\
Marble & 7 & 2 & 110.68 & 110.70 & 0.75 \\
Other & 16 & 2 & 110.29 & 110.23 & 0.67 \\
Unknown & 13 & 2 & 110.38 & 110.70 & 1.11 \\
\hline
\end{tabular}




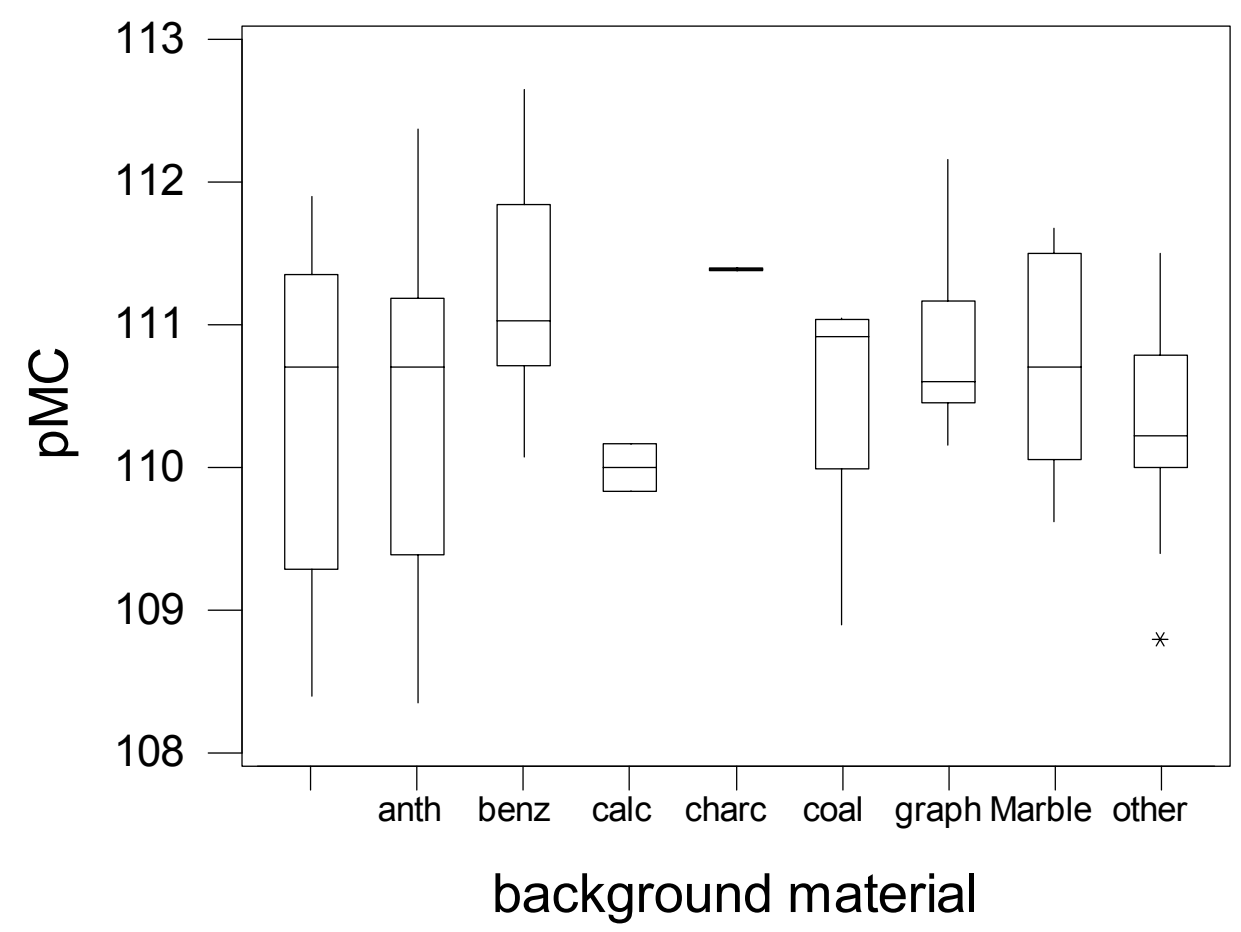

Figure 4.14a Distribution of activity by background material

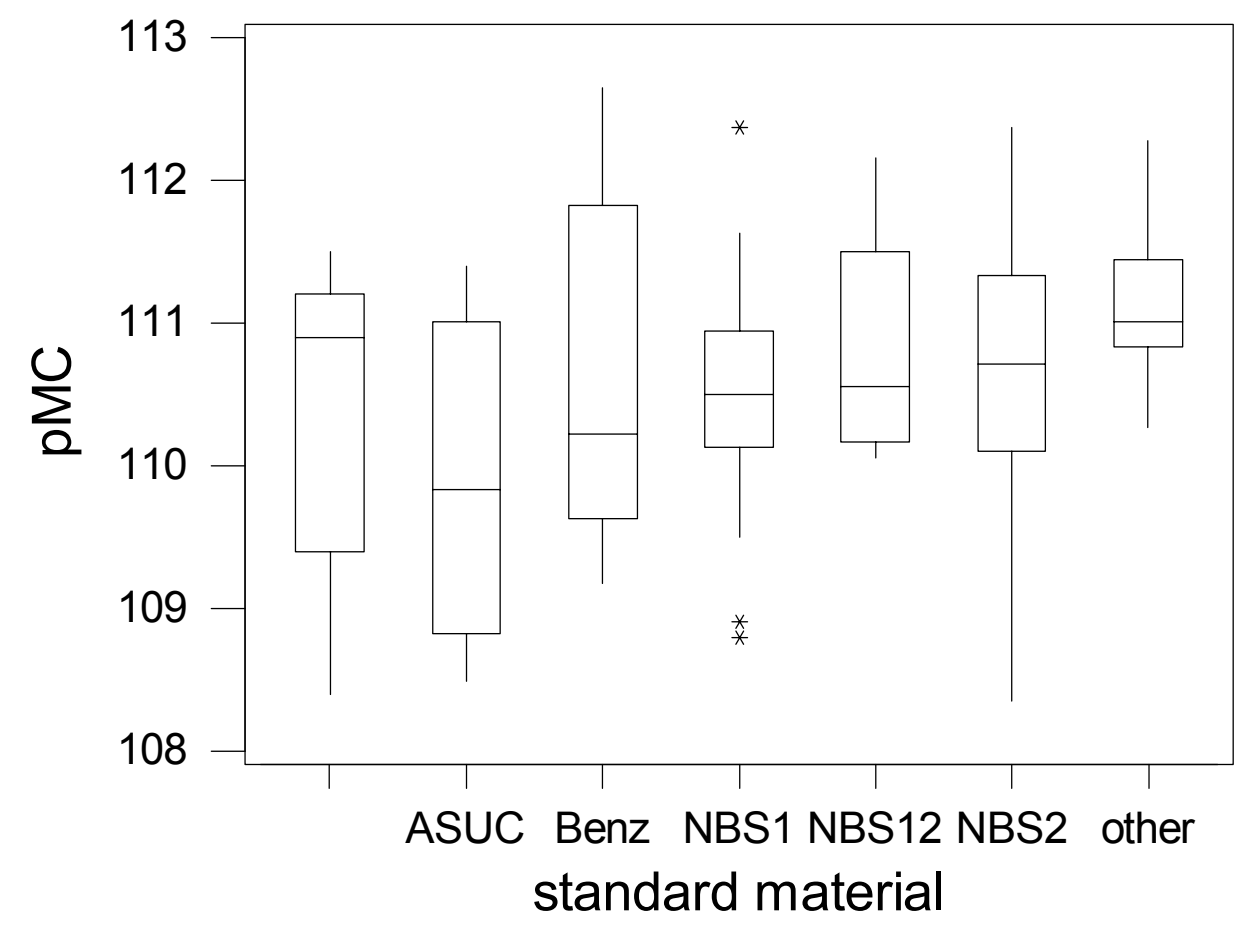

Figure $4.14 \mathrm{~b}$ Distribution of activity by modern standard 


\subsubsection{Formal Analysis}

Table 4.30a Analysis of variance of activity by modern standard

\begin{tabular}{|c|c|c|c|c|}
\hline Source & $\mathrm{DF}$ & SS & MS & F \\
\hline Standard & 5 & 4.711 & 0.942 & 0.329 \\
\hline Error & 74 & 59.228 & 0.800 & \\
\hline \multirow[t]{2}{*}{ Total } & 79 & 63.939 & & \\
\hline & & & & $\begin{array}{l}\text { Individual 95\% CIs For Mean } \\
\text { Based on Pooled StDev }\end{array}$ \\
\hline Level & $\mathrm{N}$ & Mean & StDev & ---------+----------+----------+--------- \\
\hline ASUC & 4 & 109.888 & 1.190 & $(-----------\star----------)$ \\
\hline Benz & 5 & 110.626 & 1.303 & $(----------\star----------)$ \\
\hline NBS 1 & 27 & 110.520 & 0.771 & $(----\star---)$ \\
\hline NBS12 & 8 & 110.768 & 0.781 & $(-------\star------)$ \\
\hline NBS 2 & 28 & 110.643 & 0.983 & $(---\star---)$ \\
\hline other & 8 & 111.133 & 0.586 & $(-------\star-------)$ \\
\hline Pooled St & $e v=$ & 0.895 & & 111.20 \\
\hline
\end{tabular}

Table 4.30b Analysis of variance of activity by background material

\begin{tabular}{|c|c|c|c|c|}
\hline Source & $\mathrm{DF}$ & SS & MS & F \\
\hline background & 7 & 9.538 & 1.363 & 0.079 \\
\hline Error & 66 & 46.641 & 0.707 & \\
\hline \multirow[t]{2}{*}{ Total } & 73 & 56.179 & & \\
\hline & & & & $\begin{array}{l}\text { Individual 95\% CIs For Mean } \\
\text { Based on Pooled StDev }\end{array}$ \\
\hline Level & $\mathrm{N}$ & Mean & StDev & ----+---------+---------+---------+-- \\
\hline anth & 17 & 110.447 & 1.154 & $(--\star---)$ \\
\hline benz & 13 & 111.226 & 0.797 & $(---\star---)$ \\
\hline calc & 2 & 110.000 & 0.240 & $(---------\star----------)$ \\
\hline charc & 2 & 111.390 & 0.014 & $(---------\star----------)$ \\
\hline coal & 7 & 110.513 & 0.807 & $(----\star----)$ \\
\hline graph & 10 & 110.849 & 0.643 & $(----\star---)$ \\
\hline Marble & 7 & 110.676 & 0.746 & $(----\star-----)$ \\
\hline other & 16 & 110.291 & 0.668 & $(--\star---)$ \\
\hline Pooled StD & $\mathrm{ev}=$ & 0.841 & & 112.8 \\
\hline
\end{tabular}

\subsubsection{Conclusions}

The background material is statistically significant at the $10 \%$ level, but the modern standard is not a statistically significant factor. 


\subsubsection{Sample H: Hohenheim Wood}

Table 4.31a Descriptive statistics: age by background material

\begin{tabular}{lrlllr}
\hline Background & N & Number omitted & Mean & Median & StDev \\
\hline Anth & 15 & 4 & 2254.3 & 2240.0 & 74.9 \\
Benz & 12 & 6 & 222.3 & 2249.0 & 102.6 \\
Calc & 2 & 0 & 2302.5 & 2302.5 & 53.0 \\
Charc & 2 & 0 & 2230.0 & 2230.0 & 14.1 \\
Coal & 9 & 0 & 2204.6 & 2210.0 & 52.3 \\
Graph & 10 & 0 & 2215.7 & 2215.0 & 62.9 \\
Marble & 8 & 0 & 2210.3 & 2205.0 & 118.4 \\
Other & 16 & 2 & 2233.6 & 2240.0 & 87.0 \\
Unknown & 14 & 2 & 2228.2 & 2209.5 & 66.2 \\
\hline
\end{tabular}

Table $4.31 \mathrm{~b}$ Descriptive statistics: age by modern standard

\begin{tabular}{lrlllr}
\hline Standard & N & Number omitted & Mean & Median & StDev \\
\hline ASUC & 6 & 0 & 2276.0 & 2280.0 & 127.0 \\
Benz & 4 & 2 & 2175.8 & 2133.0 & 155.0 \\
NBS1 & 27 & 2 & 2211.7 & 2210.0 & 67.5 \\
NBS12 & 8 & 0 & 2219.9 & 2225.0 & 33.4 \\
NBS2 & 28 & 7 & 2240.1 & 2240.0 & 77.7 \\
Other & 7 & 1 & 2282.4 & 2280.0 & 79.2 \\
Unknown & 8 & 2 & 2203.3 & 2190.0 & 43.7 \\
\hline
\end{tabular}

4.3.7.2 Formal Analysis

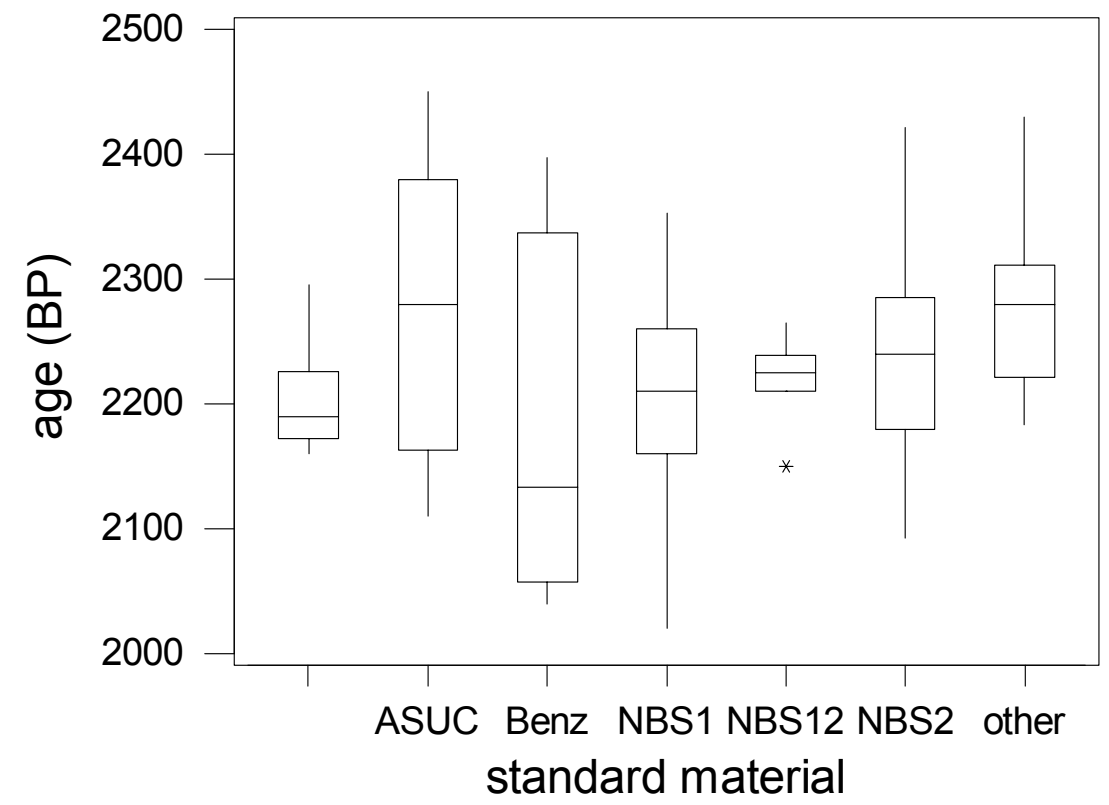

Figure 4.15a Distribution of age by modern standard 


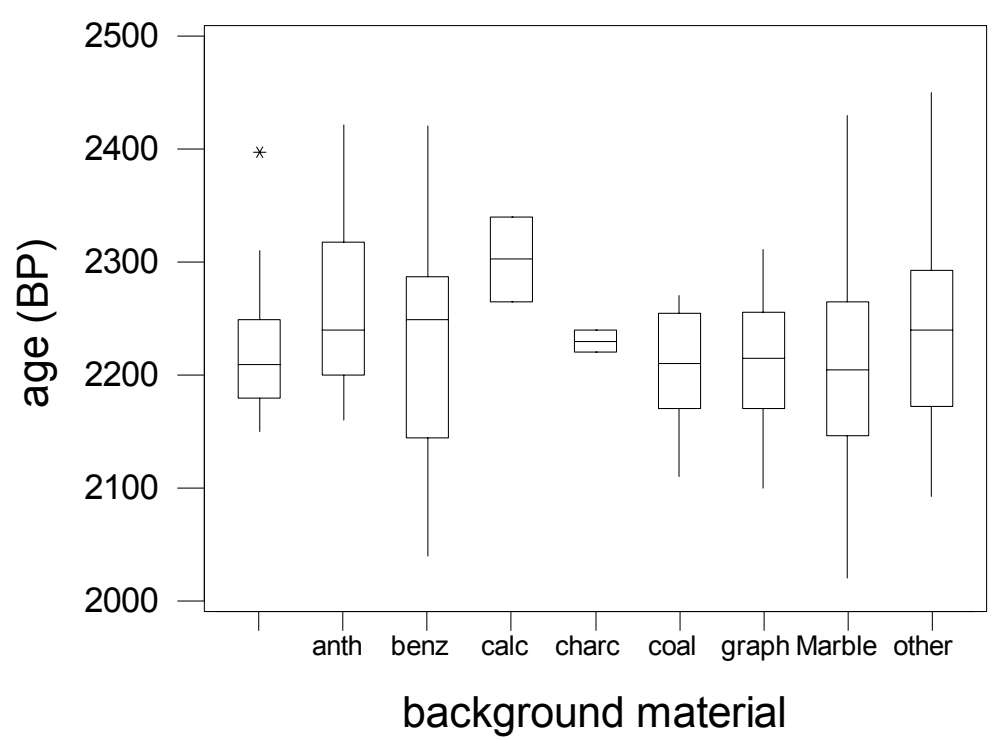

Figure 4.15b Distribution of age by background material

Table 4.32a Analysis of variance of age by background material

\begin{tabular}{|c|c|c|c|c|}
\hline Source & $\mathrm{DF}$ & SS & MS & $\mathrm{P}$ \\
\hline background & 7 & 31284 & 4469 & 0.728 \\
\hline Error & 66 & 466699 & 7071 & \\
\hline \multirow[t]{2}{*}{ Total } & 73 & 497983 & & \\
\hline & & & & $\begin{array}{l}\text { Individual 95\% CIs For Mean } \\
\text { Based on Pooled StDev }\end{array}$ \\
\hline Level & $\mathrm{N}$ & Mean & StDev & ----------+----------++----------+-------- \\
\hline anth & 15 & 2254.3 & 74.9 & $(---\star----)$ \\
\hline benz & 12 & 2222.3 & 102.6 & $(----\star----)$ \\
\hline calc & 2 & 2302.5 & 53.0 & $(-----------\star-----------)$ \\
\hline charc & 2 & 2230.0 & 14.1 & $(-----------\star-----------)$ \\
\hline coal & 9 & 2204.6 & 52.3 & $(----\star-----)$ \\
\hline graph & 10 & 2215.7 & 62.9 & $(-----\star----)$ \\
\hline Marble & 8 & 2210.3 & 118.4 & $(-----\star-----)$ \\
\hline other & 16 & 2233.6 & 87.0 & $(---\star----)$ \\
\hline Pooled StD & $\mathrm{ev}=$ & 84.1 & & 2300 \\
\hline
\end{tabular}

Table 4.32b Analysis of variance of age by modern standard

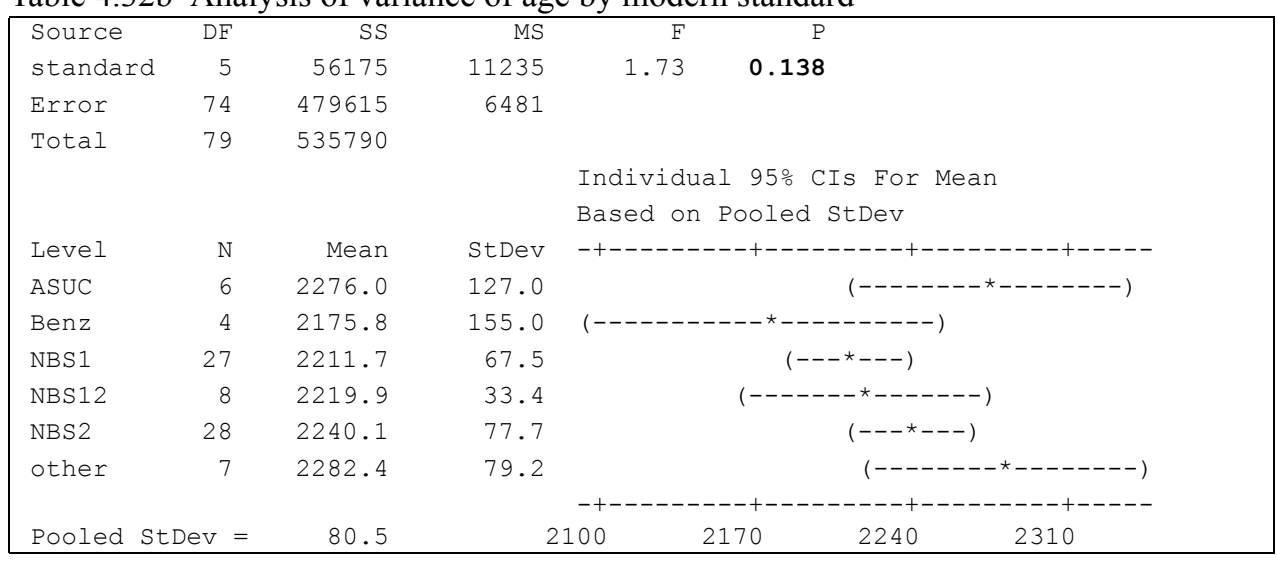




\subsubsection{Conclusions}

Again, neither standard nor background materials are statistically significant.

\subsubsection{Sample I: Belfast Cellulose}

Table 4.33a Descriptive statistics: age by modern standard

\begin{tabular}{lrlllc}
\hline Standard & N & Number omitted & Mean & Median & StDev \\
\hline ASUC & 6 & 2 & 4568.7 & 4555.0 & 120.9 \\
Benz & 4 & 1 & 4495 & 4530 & 201 \\
NBS1 & 25 & 3 & 4473.5 & 4468.0 & 73.2 \\
NBS12 & 8 & 0 & 4490.8 & 4490.0 & 53.9 \\
NBS2 & 31 & 3 & 4500.8 & 4500.0 & 116.7 \\
Other & 6 & 2 & 4500.5 & 4455.5 & 88.6 \\
Unknown & 6 & 3 & 4431.7 & 4430.0 & 51.3 \\
\hline
\end{tabular}

Table 4.33b Descriptive statistics: age by background material

\begin{tabular}{lrllll}
\hline Background & $\mathrm{N}$ & $\mathrm{N}^{*}$ & Mean & Median & StDev \\
\hline Anth & 16 & 1 & 4483.3 & 4490.0 & 111.7 \\
Benz & 15 & 4 & 4523.5 & 4520.0 & 156.3 \\
Calc & 2 & 0 & 4540.0 & 4540.0 & 56.6 \\
Charc & 2 & 0 & 4420.0 & 4420.0 & 14.1 \\
Coal & 6 & 2 & 4485.0 & 4490.0 & 53.9 \\
Graph & 11 & 0 & 4458.6 & 4461.0 & 87.5 \\
Marble & 6 & 2 & 4474.0 & 4480.0 & 76.8 \\
Other & 16 & 3 & 4513.0 & 4495.0 & 82.4 \\
Unknown & 12 & 2 & 4480.2 & 4485.0 & 75.9 \\
\hline
\end{tabular}

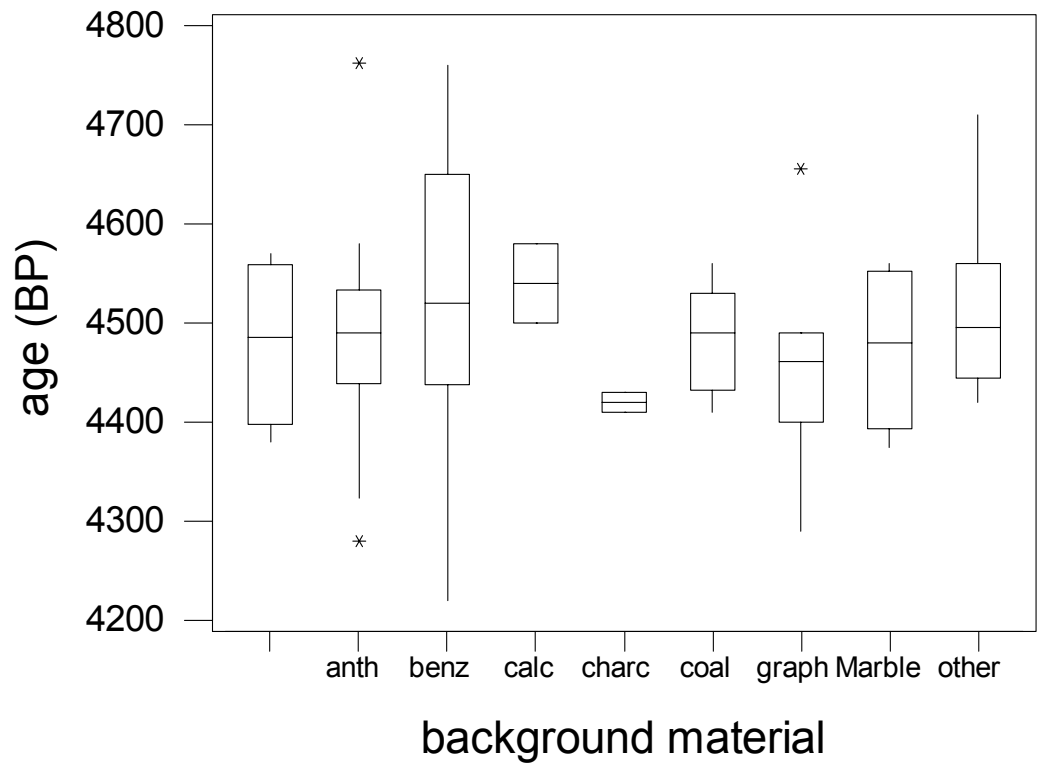

Figure 4.16a Distribution of age by background material 


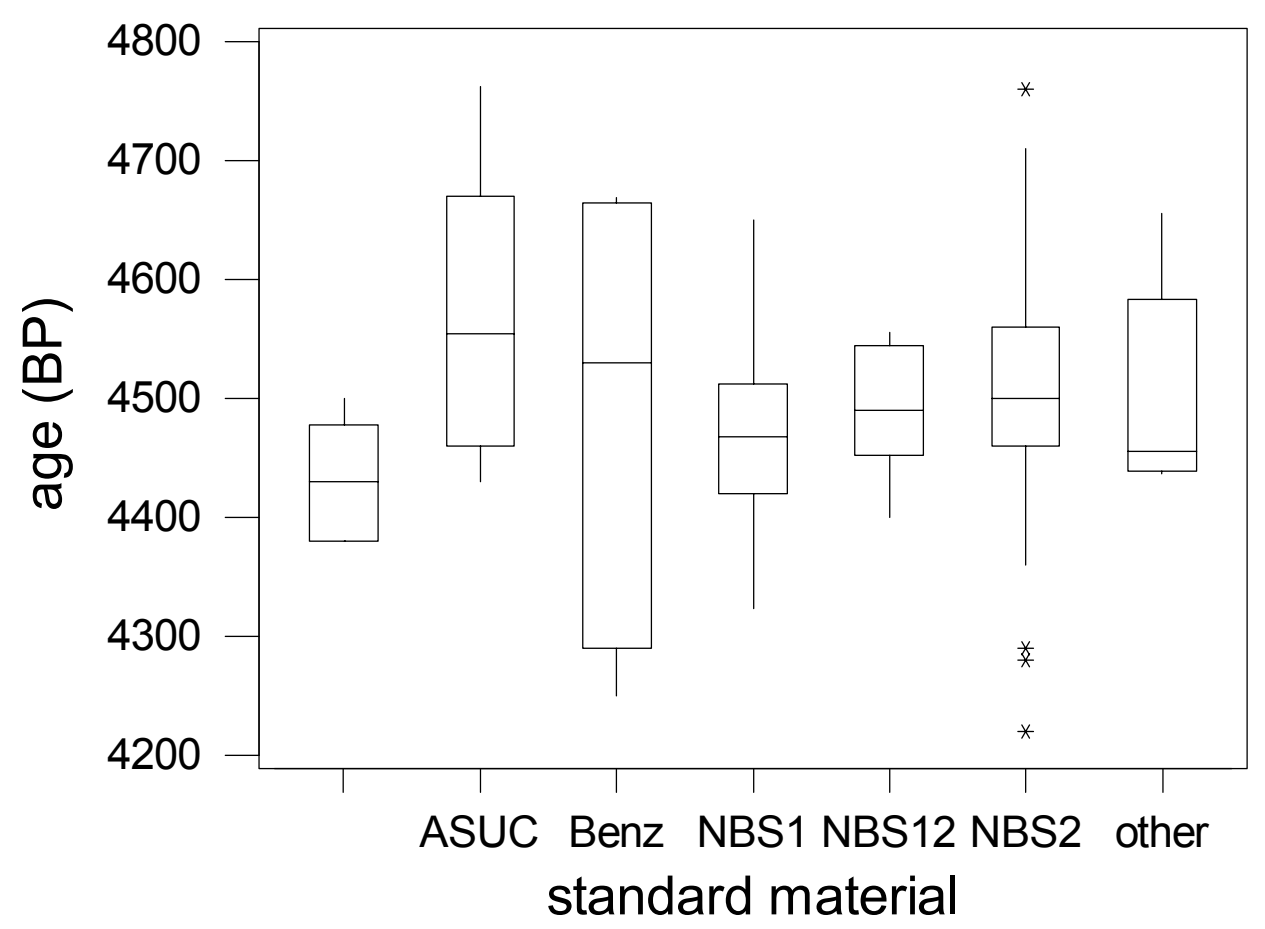

Figure 4.16b Distribution of age by modern standard

\subsubsection{Formal analysis}

Table 4.34a Analysis of variance of age by modern standard

\begin{tabular}{|c|c|c|c|c|}
\hline Source & $\mathrm{DF}$ & SS & MS & $\mathrm{P}$ \\
\hline standard & 5 & 45428 & 9086 & 0.519 \\
\hline Error & 74 & 791207 & 10692 & \\
\hline \multirow[t]{2}{*}{ Total } & 79 & 836635 & & \\
\hline & & & & $\begin{array}{l}\text { Individual 95\% CIs For Mean } \\
\text { Based on Pooled StDev }\end{array}$ \\
\hline Level & $\mathrm{N}$ & Mean & StDev & --+---------+---------++---------+---- \\
\hline ASUC & 6 & 4568.7 & 120.9 & $(---------\star----------)$ \\
\hline Benz & 4 & 4494.8 & 201.3 & $(------------\star------------)$ \\
\hline NBS 1 & 25 & 4473.5 & 73.2 & $(----\star----)$ \\
\hline NBS 12 & 8 & 4490.8 & 53.9 & $(--------\star--------)$ \\
\hline NBS 2 & 31 & 4500.8 & 116.7 & $(----\star---)$ \\
\hline other & 6 & 4500.5 & 88.6 & $(----------\star---------)$ \\
\hline Pooled St & $e v=$ & 103.4 & & 4640 \\
\hline
\end{tabular}

\subsubsection{Conclusions}

The background and standard materials are not statistically significant factors in explaining the observed variation. 
Table 4.34b Analysis of variance of age by background material

\begin{tabular}{|c|c|c|c|c|}
\hline Source & $\mathrm{DF}$ & SS & $\mathrm{MS}$ & F \\
\hline background & 7 & 52502 & 7500 & 0.709 \\
\hline Error & 66 & 755202 & 11442 & \\
\hline \multirow[t]{2}{*}{ Total } & 73 & 807704 & & \\
\hline & & & & $\begin{array}{l}\text { Individual 95\% CIs For Mean } \\
\text { Based on Pooled StDev }\end{array}$ \\
\hline Level & $\mathrm{N}$ & Mean & StDev & -----+---------+---------+---------+ \\
\hline anth & 16 & 4483.3 & 111.7 & $(----\star---)$ \\
\hline benz & 15 & 4523.5 & 156.3 & $(----\star----)$ \\
\hline calc & 2 & 4540.0 & 56.6 & $(-----------\star------------)$ \\
\hline charc & 2 & 4420.0 & 14.1 & $---\star------------)$ \\
\hline coal & 6 & 4485.0 & 53.9 & $(-------\star------)$ \\
\hline graph & 11 & 4458.6 & 87.5 & $(-----\star----)$ \\
\hline Marble & 6 & 4474.0 & 76.8 & $(------\star------)$ \\
\hline other & 16 & 4513.0 & 82.4 & $(---\star----)$ \\
\hline Pooled StDe & $\mathrm{ev}=$ & 107.0 & & 4560 \\
\hline
\end{tabular}

\subsection{SUMMARY FINDINGS OF BACKGROUND AND STANDARD MATERIAL EFFECTS}

The information from this analysis is summarized in the table below. With few exceptions, after omission of outliers, the background and standard material is not a significant factor in explaining the observed variation. A preliminary analysis, before omission of the outliers, had however shown that standard was often a significant factor. Thus, one inference from this is that the modern standard is an important factor, but that it may be an indirect measure of the laboratory capability and experience and may be related to the presence of outliers.

Table 4.35 Summary of analysis of variance findings ${ }^{\mathrm{a}}$

\begin{tabular}{lll}
\hline FIRI sample & Background & Standard \\
\hline A $($ pmC) & S & S \\
B $($ pmC) & S $(10 \%)$ & S \\
C & NS & NS \\
D & NS & S \\
E & NS & NS \\
F & NS & NS \\
G & NS & NS \\
H & NS & NS \\
I & NS & NS \\
J & S $(10 \%)$ & NS \\
${ }^{a}$ S = significant; NS = not significant & &
\end{tabular}

\subsection{PRETREATMENT AS A FACTOR IN EXPLAINING THE VARIATION}

Pretreatment may also be a source of variation, but it should only be appropriate in a few samples, specifically the whole wood and the turbidite. The following tables enumerate the numbers of laboratories using a specific or general method for the samples. It also contains information pertinent to the issue of the explanation for outliers. Not all laboratories reported this information, so the table is incomplete. 
Table 4.36 Number of laboratories (and laboratories with outliers) using specified pretreatment methods for Samples D, F, H, and I

\begin{tabular}{|c|c|c|c|c|c|}
\hline \multicolumn{3}{|c|}{$\begin{array}{l}\text { Samples D, F, and H } \\
\text { (Wood) }\end{array}$} & \multicolumn{3}{|c|}{$\begin{array}{l}\text { Sample I } \\
\text { (Cellulose) }\end{array}$} \\
\hline Method & $\begin{array}{l}\text { Non-outlier } \\
\text { laboratories }\end{array}$ & $\begin{array}{l}\text { All } \\
\text { laboratories }\end{array}$ & Method & $\begin{array}{l}\text { Non-outlier } \\
\text { laboratories }\end{array}$ & $\begin{array}{l}\text { All } \\
\text { laboratories }\end{array}$ \\
\hline AAA & 40 & 41 & AAA & 1 & 1 \\
\hline AAAA & 2 & 2 & Missing & 10 & 13 \\
\hline Cellulose & 14 & 14 & None & 56 & 62 \\
\hline Missing & 11 & 11 & Other & 9 & 11 \\
\hline Other & 4 & 5 & Total & 76 & 87 \\
\hline None & 4 & 4 & & & \\
\hline More than 1 & 8 & 8 & & & \\
\hline Total & 83 & 85 & & & \\
\hline
\end{tabular}

Table 4.37 Number of laboratories (and laboratories with outliers) using specified pretreatment methods for Sample C and Sample E

\begin{tabular}{|c|c|c|c|c|c|}
\hline \multicolumn{3}{|c|}{$\begin{array}{c}\text { Sample C } \\
\text { (Turbidite) }\end{array}$} & \multicolumn{3}{|c|}{$\begin{array}{c}\text { Sample E } \\
\text { (Humic acid) }\end{array}$} \\
\hline Method & $\begin{array}{l}\text { Non-outlier } \\
\text { laboratories }\end{array}$ & $\begin{array}{l}\text { All } \\
\text { laboratories }\end{array}$ & Method & $\begin{array}{l}\text { Non-outlier } \\
\text { laboratories }\end{array}$ & $\begin{array}{l}\text { All } \\
\text { laboratories }\end{array}$ \\
\hline Acid leaching & 5 & 7 & AAA & 1 & 1 \\
\hline Missing & 10 & 11 & Missing & 6 & 11 \\
\hline None & 54 & 69 & None & 53 & 59 \\
\hline Other & 1 & 2 & Other & 8 & 11 \\
\hline Total & 70 & 79 & Total & 68 & 82 \\
\hline
\end{tabular}

Table 4.38 Number of laboratories (and laboratories with outliers) using specified pretreatment methods for Samples G and J

\begin{tabular}{lcc}
\hline \multicolumn{2}{c}{$\begin{array}{c}\text { Sample G and J } \\
\text { (barley mash) }\end{array}$} \\
\hline Method & Non-outlier laboratories & All laboratories \\
\hline AAA & 5 & 7 \\
Missing & 10 & 11 \\
None & 54 & 69 \\
Other & 1 & 2 \\
Total & $\mathbf{7 0}$ & $\mathbf{7 9}$ \\
\hline
\end{tabular}

Table 4.39 Description of pretreatment methods classed as "other" for each sample type

\begin{tabular}{clllll}
\hline Sample & \multicolumn{1}{c}{$\begin{array}{l}\text { Wood } \\
(\mathrm{D}, \mathrm{F}, \text { and } \mathrm{H})\end{array}$} & $\begin{array}{c}\text { Turbidite } \\
(\mathrm{C})\end{array}$ & $\begin{array}{c}\text { Humic acid } \\
(\mathrm{E})\end{array}$ & $\begin{array}{l}\text { Barley mash } \\
(\mathrm{G} \text { and } \mathrm{J})\end{array}$ & $\begin{array}{c}\text { Cellulose } \\
(\mathrm{I})\end{array}$ \\
\hline Other methods & $\begin{array}{l}\text { Acid only } \\
\text { "Routine" }\end{array}$ & $\begin{array}{l}\text { AAA } \\
\text { "Routine" }\end{array}$ & $\begin{array}{l}\text { Burning } \\
\text { AAA } \\
\text { "Routine" }\end{array}$ & $\begin{array}{l}\text { AAA } \\
\text { "Routine" }\end{array}$ & $\begin{array}{l}\text { Burning } \\
\text { AAA } \\
\text { "Routine" }\end{array}$ \\
\hline
\end{tabular}


A variety of pretreatment methods have been used, even for Sample C (turbidite) where the instructions stated that the sample should not be pretreated. AAA was the most commonly used method, with 14 laboratories extracting cellulose from the wood samples.

\subsubsection{Sample C (Turbidite)}

Table 4.40 Descriptive statistics: age by pretreatment method

\begin{tabular}{lrlll}
\hline Pretreatment & N & Mean & Median & StDev \\
\hline Acid leach & 9 & 18,037 & 18,090 & 150 \\
Missing & 10 & 18,166 & 18,165 & 153 \\
None & 64 & 18,166 & 18,174 & 209 \\
Other & 1 & 18,359 & 18,359 & $*$ \\
\hline
\end{tabular}

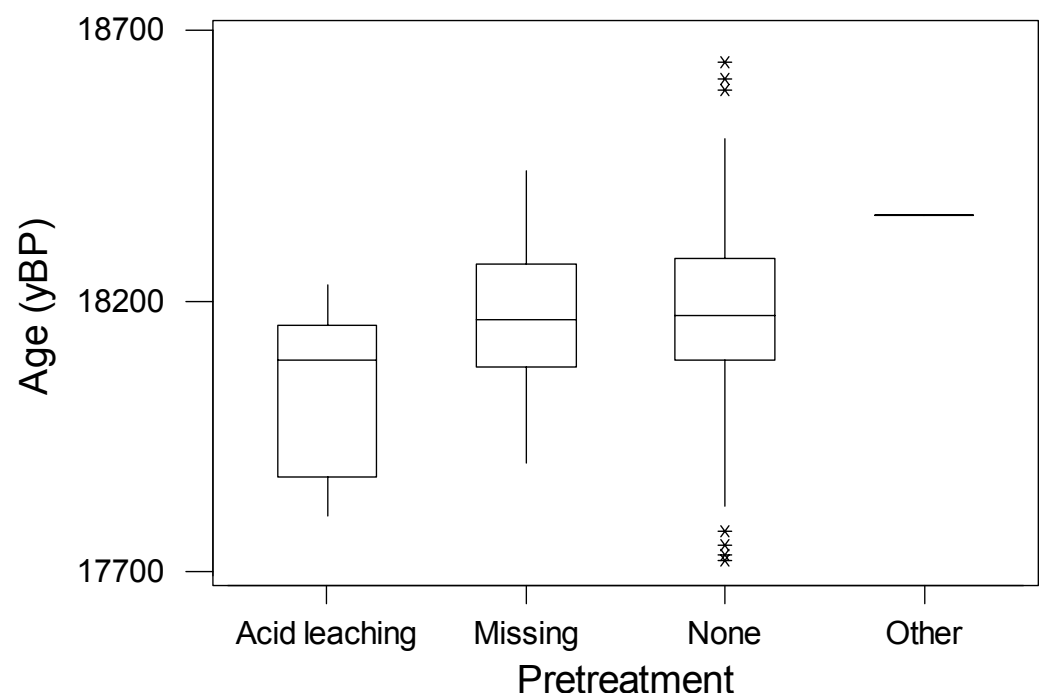

Figure 4.17 Distribution of age by pretreatment

A formal analysis to compare the results of the acid leaching and not pretreating gave a p-value of 0.04 , indicating that there was a significant difference between the acid-leached and non-pretreated results. Such a difference had already been seen in the homogeneity testing and was the reason for the instruction that this sample should not be pretreated. In this case, the acid-leached results are younger.

\subsubsection{Sample H (Hohenheim dendro-dated wood) and Samples D and F (Belfast)}

Table 4.41 Descriptive statistics: age for Sample H

\begin{tabular}{lrlll}
\hline Pretreatment & $\mathrm{N}$ & Mean & Median & StDev \\
\hline AAA & 50 & 2230.3 & 2230.0 & 86.4 \\
AAAA & 2 & 2207.5 & 2207.5 & 46.0 \\
Cell. ex & 17 & 2246.6 & 2248.0 & 77.9 \\
Missing & 11 & 2230.7 & 2209.0 & 66.4 \\
None & 4 & 2157.5 & 2170.0 & 33.0 \\
Other & 4 & 2215.5 & 2195.0 & 72.4 \\
\hline
\end{tabular}




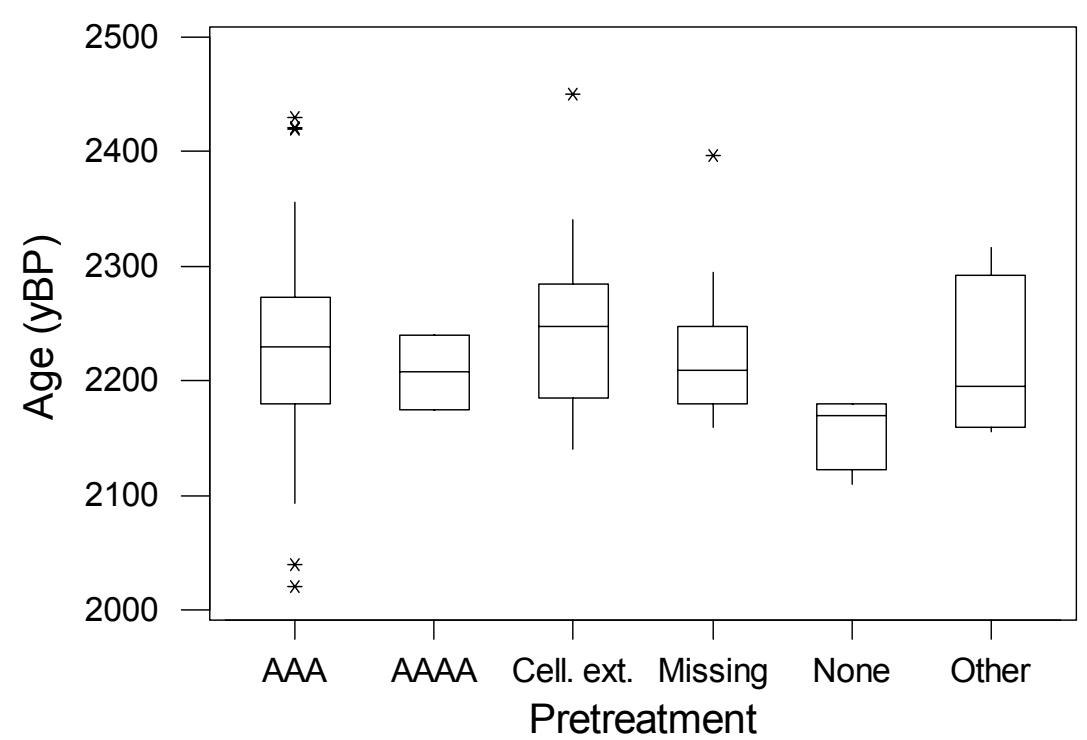

Figure 4.18 Distribution of age by pretreatment

Table 4.42 Descriptive statistics for age of Samples D and F

\begin{tabular}{lrrll}
\hline Pretreatment & $\mathrm{N}$ & Mean & Median & StDev \\
\hline AAA & 100 & 4523.3 & 4510.5 & 73.1 \\
AAAA & 4 & 4468.5 & 4472.5 & 16.1 \\
Cell. ex & 43 & 4533.1 & 4540.0 & 73.7 \\
Missing & 25 & 4488.1 & 4493.0 & 92.6 \\
None & 4 & 4468.8 & 4477.5 & 37.1 \\
Other & 9 & 504.1 & 4505.0 & 91.3 \\
\hline
\end{tabular}

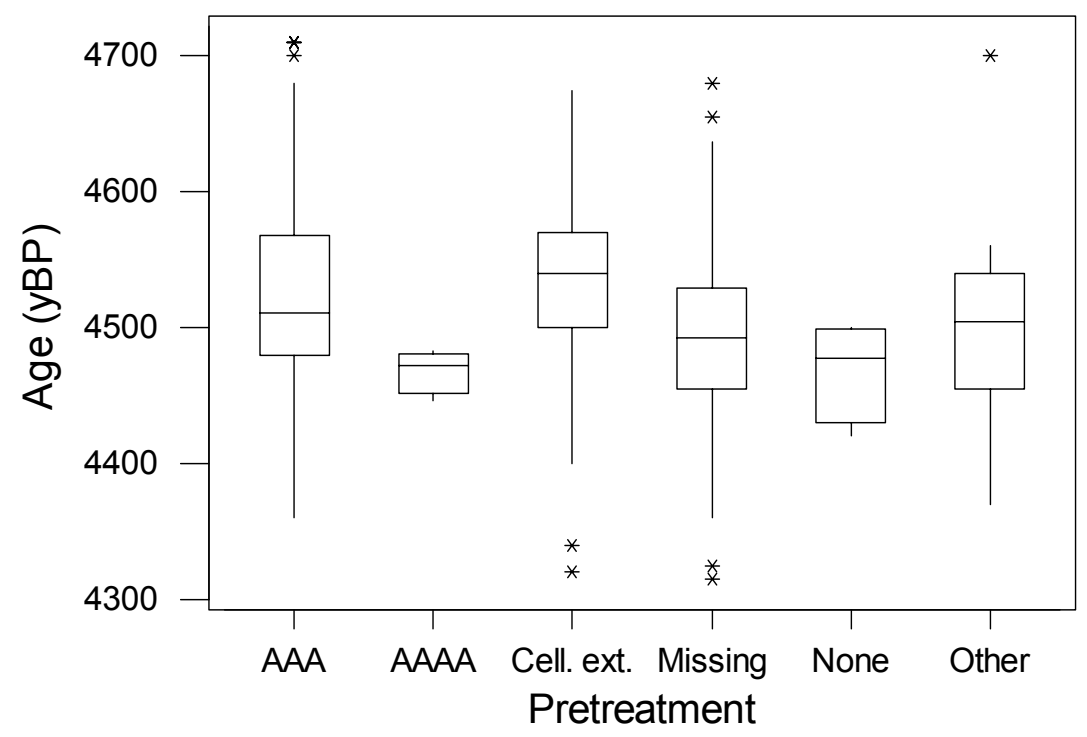

Figure 4.19 Distribution of age by pretreatment method 


\subsubsection{Samples $D, F$, and $H$ (Wood)}

Table 4.43 Descriptive statistics of deviation from consensus for all wood samples by pretreatment method

\begin{tabular}{lrcccl}
\hline Pretreatment & \multicolumn{1}{c}{$\mathrm{N}$} & \multicolumn{1}{c}{ Mean } & Median & TrMean & StDev \\
\hline AAA & 150 & -0.0586 & -0.0387 & -0.0531 & 0.6407 \\
AAAA & 6 & 0.2604 & 0.2663 & 0.2604 & 0.1952 \\
Cell. ex & 60 & -0.1740 & -0.1651 & -0.1749 & 0.5594 \\
Missing & 36 & 0.119 & 0.125 & 0.135 & 0.643 \\
None & 9 & 0.447 & 0.506 & 0.447 & 0.358 \\
Other & 13 & 0.085 & 0.164 & 0.129 & 0.630 \\
\hline
\end{tabular}

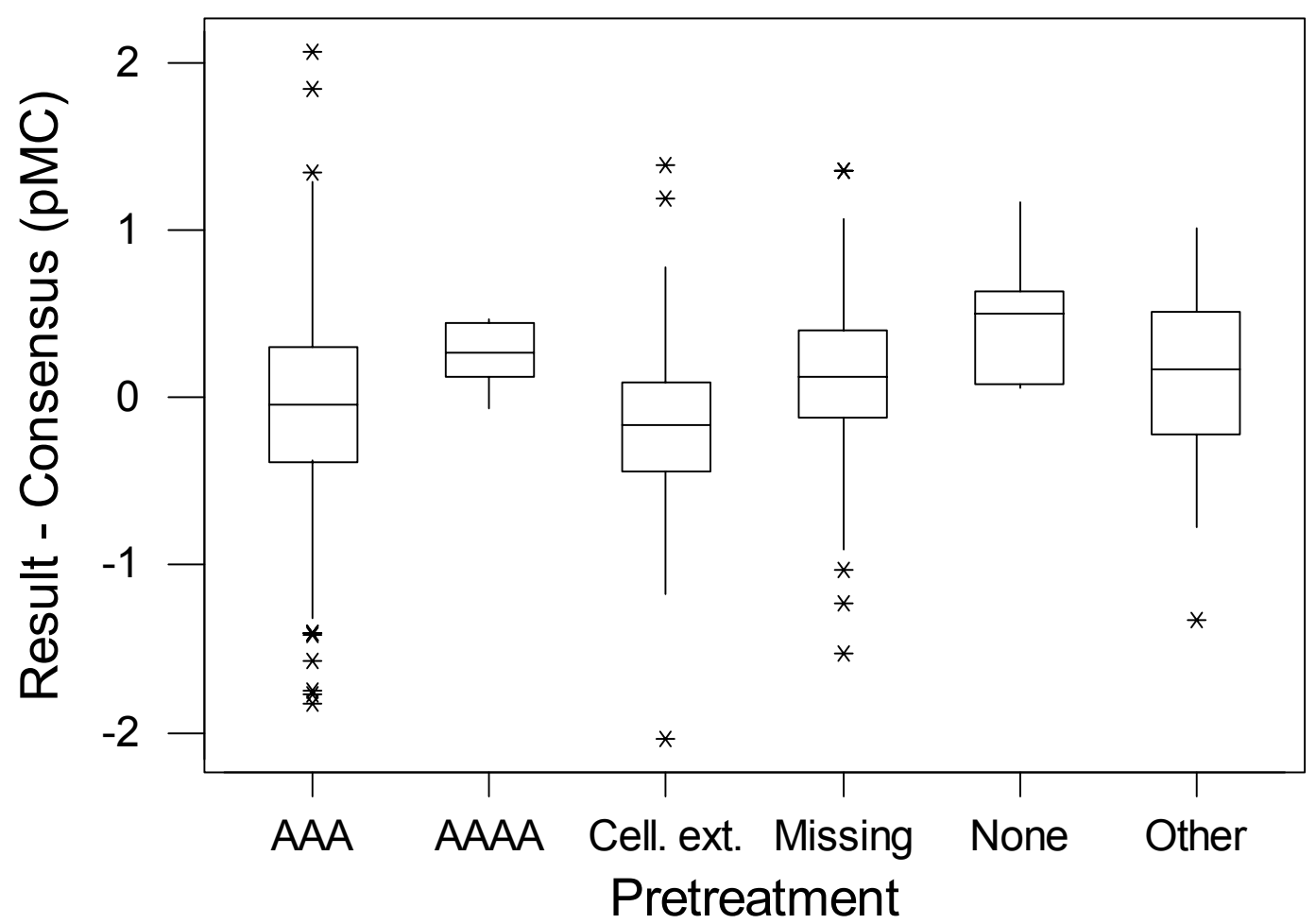

Figure 4.20 Distribution of deviation from consensus by pretreatment method

To use all the wood samples together, we have centered each sample at the consensus value (see Section 7) and investigated if method of pretreatment may be a source of variation. 


\subsubsection{Formal Analysis}

Table 4.44 Analysis of variance of deviation from consensus by pretreatment method

\begin{tabular}{|c|c|c|c|c|}
\hline Source & $\overline{\mathrm{DF}}$ & SS & MS & F \\
\hline Pretreat & 5 & 4.894 & 0.979 & 0.025 \\
\hline Error & 268 & 100.062 & 0.373 & \\
\hline \multirow[t]{2}{*}{ Total } & 273 & 104.956 & & \\
\hline & & & & $\begin{array}{l}\text { Individual 95\% CIs For Mean } \\
\text { Based on Pooled StDev }\end{array}$ \\
\hline Level & $\mathrm{N}$ & Mean & StDev & ----------+---------+-- \\
\hline AAA & 150 & -0.0586 & 0.6407 & $(-\star--)$ \\
\hline AAAA & 6 & 0.2604 & 0.1952 & $-\star \star-------------)$ \\
\hline Cell. ex & 60 & -0.1740 & 0.5594 & $(---\star---)$ \\
\hline Missing & 36 & 0.1190 & 0.6426 & $(----\star-----)$ \\
\hline None & 9 & 0.4471 & 0.3582 & $(-----------\star----------)$ \\
\hline Other & 13 & 0.0851 & 0.6299 & $(--------\star---------)$ \\
\hline Pooled St & Dev $=$ & 0.6110 & & 0.35 \\
\hline
\end{tabular}

\subsubsection{Conclusions}

A statistically significant effect of the pretreatment method was found (p-value $<0.05$ ). There is a large amount of literature about the effects of pretreatment, and specifically for wood samples. The magnitude of the effect here, while statistically significant is, in fact, of little practical importance since any differences are very small.

\subsubsection{Sample E: Humic Acid}

Table 4.45 Descriptive statistics: age by pretreatment method

\begin{tabular}{lrllc}
\hline Pretreatment & N & Mean & Median & StDev \\
\hline Missing & 6 & 11,720 & 11,756 & 98 \\
None & 61 & 11,778 & 11,778 & 143 \\
Other & 9 & 11,742 & 11,720 & 163 \\
\hline
\end{tabular}

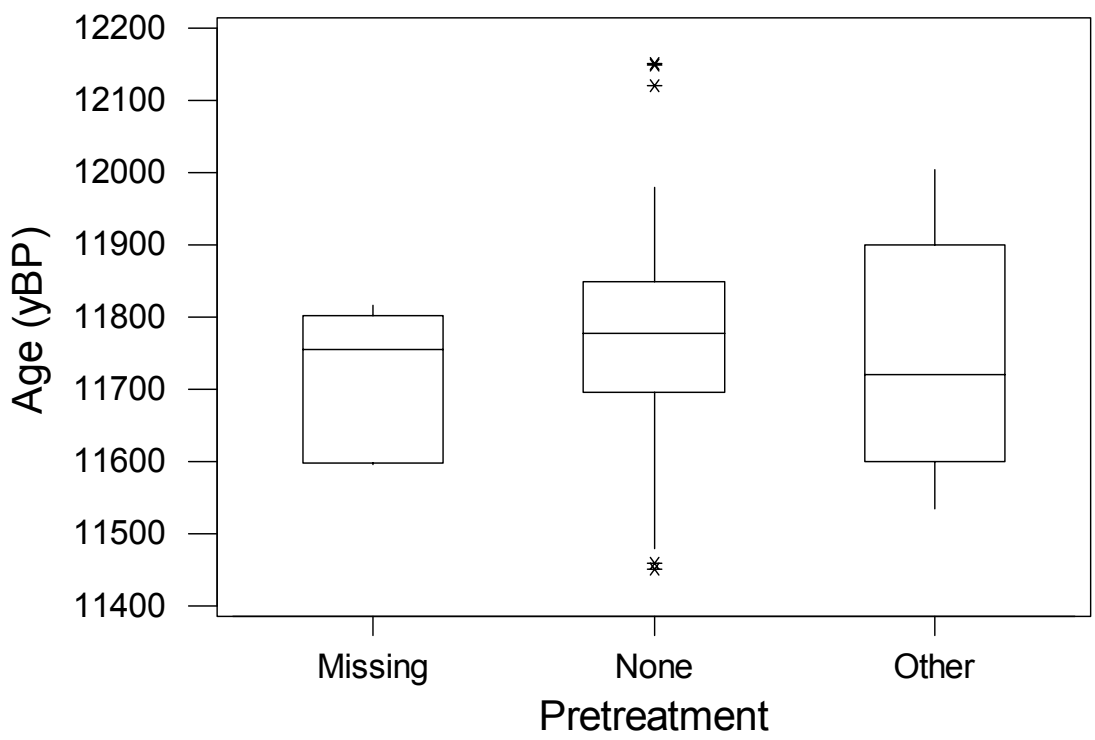

Figure 4.21 Distribution of age by pretreatment method 


\subsubsection{Formal Analysis}

Table 4.46 Analysis of variance by pretreatment method

\begin{tabular}{|c|c|c|c|c|}
\hline \multicolumn{5}{|c|}{ Analysis of Variance for Age } \\
\hline Source & $\mathrm{DF}$ & SS & MS & $\mathrm{P}$ \\
\hline Pretreat & 2 & 25789 & 12895 & 0.532 \\
\hline Error & 73 & 1479517 & 20267 & \\
\hline \multirow[t]{2}{*}{ Total } & 75 & 1505306 & & \\
\hline & & & & $\begin{array}{l}\text { Individual 95\% CIs For Mean } \\
\text { Based on Pooled StDev }\end{array}$ \\
\hline Level & $\mathrm{N}$ & Mean & StDev & ---+---------+----------+---------+--- \\
\hline Missing & 6 & 11720 & 98 & $(----------------\star-----------------)$ \\
\hline None & 61 & 11778 & 143 & $(-----\star----)$ \\
\hline Other & 9 & 11742 & 163 & $(------------\star-------------)$ \\
\hline Pooled St & $\mathrm{ev}=$ & 142 & & 11620 \\
\hline
\end{tabular}

\subsubsection{Conclusion}

No statistically significant effect of pretreatment is observed.

\subsubsection{SAMPLES G and J: Barley Mash}

Table 4.47 Descriptive statistics: pMC by pretreatment

\begin{tabular}{lrlll}
\hline Pretreatment & \multicolumn{1}{c}{ N } & Mean & Median & StDev \\
\hline AAA & 5 & 110.58 & 110.76 & 0.54 \\
Missing & 28 & 110.64 & 110.83 & 0.87 \\
None & 120 & 110.61 & 110.64 & 0.90 \\
Other & 18 & 110.52 & 110.50 & 1.24 \\
\hline
\end{tabular}

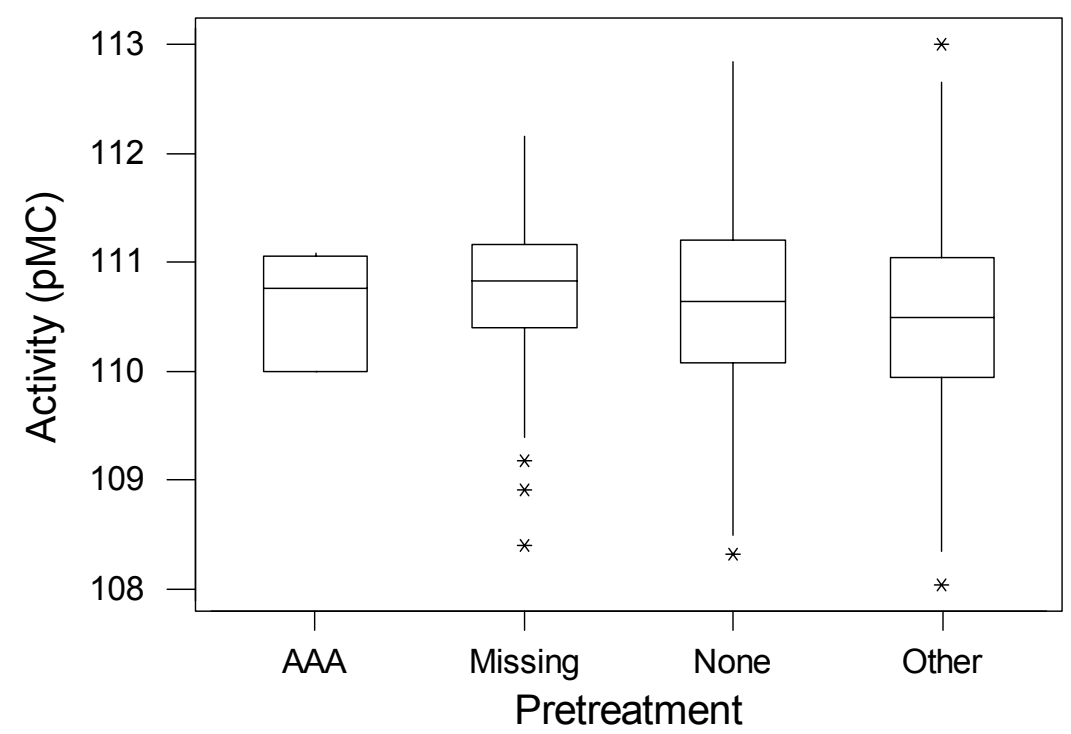

Figure 4.22 Distribution of activity by pretreatment method 


\subsubsection{Formal Analysis}

Table 4.48 Analysis of variance of activity by pretreatment method

\begin{tabular}{|c|c|c|c|c|}
\hline \multicolumn{5}{|c|}{ Analysis of Variance for pMC } \\
\hline Source & $\mathrm{DF}$ & SS & MS & $\mathrm{P}$ \\
\hline Pretreat & 3 & 0.159 & 0.053 & 0.980 \\
\hline Error & 167 & 145.479 & 0.871 & \\
\hline \multirow[t]{2}{*}{ Total } & 170 & 145.638 & & \\
\hline & & & & $\begin{array}{l}\text { Individual 95\% CIs For Mean } \\
\text { Based on Pooled StDev }\end{array}$ \\
\hline Level & $\mathrm{N}$ & Mean & StDev & -----+---------+---------+---------+- \\
\hline AAA & 5 & 110.576 & 0.540 & $(-----------------\star----------------)$ \\
\hline Missing & 28 & 110.638 & 0.873 & $(------\star------)$ \\
\hline None & 120 & 110.612 & 0.905 & $(--\star---)$ \\
\hline Other & 18 & 110.524 & 1.244 & $(--------\star--------)$ \\
\hline Pooled St & Dev $=$ & 0.933 & & 111.00 \\
\hline
\end{tabular}

\subsubsection{Conclusion}

No statistically significant effect of pretreatment is observed.

\subsubsection{SAMPLE I: Belfast Cellulose}

Table 4.49 Descriptive statistics: age by pretreatment

\begin{tabular}{llllc}
\hline Pretreatment & N & Mean & Median & StDev \\
\hline Missing & 13 & 4446.0 & 4452.0 & 54.6 \\
None & 63 & 4505.7 & 4490.0 & 91.8 \\
Other & 10 & 4463.5 & 4435.0 & 171.7 \\
\hline
\end{tabular}

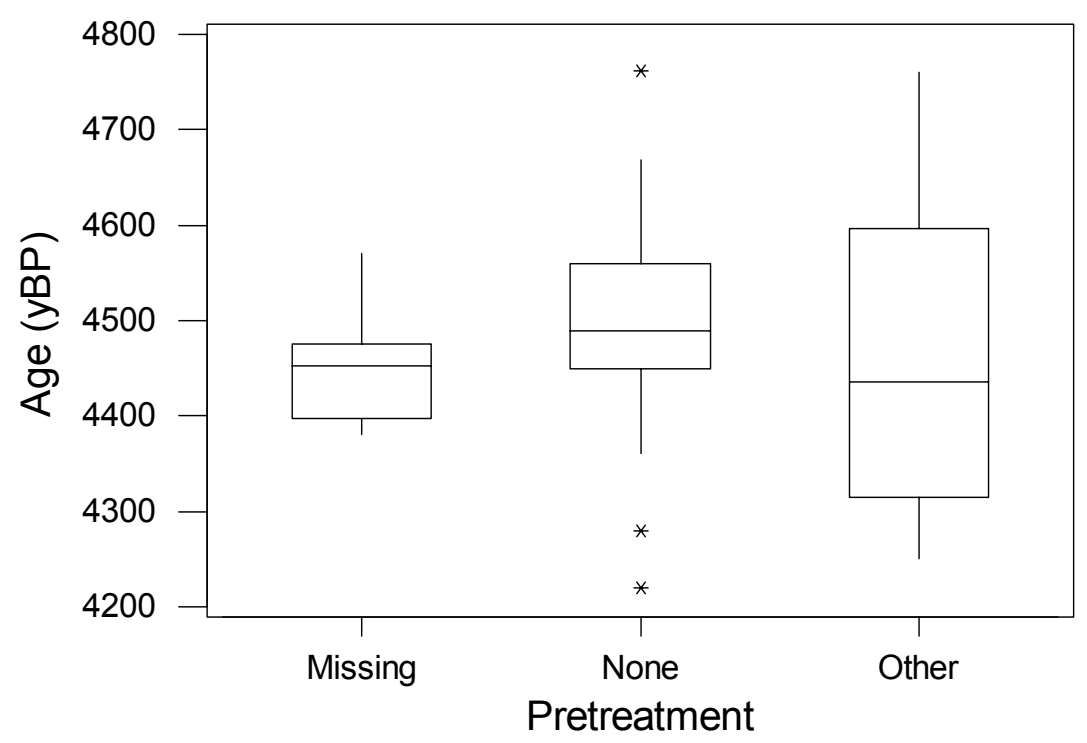

Figure 4.23 Distribution of age by pretreatment method 


\subsubsection{Formal Analysis}

Table 4.50 Analysis of variance of age by pretreatment method

\begin{tabular}{|c|c|c|c|c|}
\hline \multicolumn{5}{|c|}{ Analysis of Variance for Age } \\
\hline Source & $\mathrm{DF}$ & SS & MS & $\mathrm{P}$ \\
\hline Pretreat & 2 & 47398 & 23699 & 0.098 \\
\hline Error & 83 & 823879 & 9926 & \\
\hline \multirow[t]{2}{*}{ Total } & 85 & 871278 & & \\
\hline & & & & $\begin{array}{l}\text { Individual 95\% CIs For Mean } \\
\text { Based on Pooled StDev }\end{array}$ \\
\hline Level & $\mathrm{N}$ & Mean & StDev & ---+----------+----------+-----------+--- \\
\hline Missing & 13 & 4446.0 & 54.6 & $(-------------\star------------)$ \\
\hline None & 63 & 4505.7 & 91.8 & $(-----\star------)$ \\
\hline Other & 10 & 4463.5 & 171.7 & $(---------------\star---------------)$ \\
\hline Pooled S & $e v=$ & 99.6 & & 4400 \\
\hline
\end{tabular}

\subsubsection{Conclusion}

A statistically significant effect of pretreatment at $10 \%$ is observed, but given the insufficient information provided by the laboratories, no further conclusions can be drawn for this sample. 